\title{
LA ESTELA DE LAS INGENIERAS DOMÉSTICAS AMERICANAS EN LA VIVIENDA SOCIAL EUROPEA
} THE TRAIL OF AMERICAN DOMESTIC ENGINEERS IN EUROPEAN SOCIAL HOUSING

Carmen Espegel Alonso; Gustavo Rojas Pérez

RESUMEN El presente artículo analiza la gran influencia que los Manuales escritos por las norteamericanas Catharine Beecher, Christine Frederick y Lillian Gilbreth tuvieron sobre la concepción de la domesticidad en los programas residenciales de la Nueva Oficina de Construcción de Frankfurt, liderada por Ernst May entre 1925 y 1930, en la que Margarete Schütte-Lihotzky se encargó del estudio de los hábitos de vida.

Desde posiciones muy diversas, como la intelectualidad de Beecher, el pragmatismo de Frederick y el cientifismo de Gilbreth, todas ellas tipificaron, hicieron eficiente y optimizaron, respectivamente, la concepción proyectual y productiva de la vivienda. Esto implicaba racionalizar, modernizar y mecanizar su contenido con el fin de mejorar el trabajo del ama de casa. En sus textos, no se cuestiona si el lugar apropiado para la mujer es el hogar, sino que se afirma que no está bien ideado. Por primera vez, la casa es pensada desde un nuevo punto de vista: el del usuario.

A través del artículo se revelan, desde un punto de vista no planteado anteriormente, algunos de los posibles medios de transmisión de las investigaciones americanas al contexto europeo.

PALABRAS CLAVE vivienda colectiva; domesticidad; racionalización; mecanización; cocina; ingenieras domésticas

SUMMARY This article analyzes the great influence that the Manuals written by Catharine Beecher, Christine Frederick and Lillian Gilbreth had in the conception of domesticity in the residential programs carried out by the New Frankfurt initiative led by Ernst May between 1925 and 1930, in which Margarete Schütte-Lihotzky was in charge of studying life habits.

From their different stances, Beecher's intellectualism, Frederick's pragmatism and Gilberth's scientism, they all typified, made more efficient and optimized, respectively, the design and productive conception of the house. This meant rationalizing, modernizing and mechanizing its content with the aim of improving the work conditions of the housewife. Their texts do not question whether the home is the adequate place for women, they just state that its spaces are not correctly thought out. For the very first time, the house was conceived from a new perspective: that of the user.

This article reveals, from a novel point of view, some of the possible means by which the research carried out by these American women reached the European context.

KEY WORDS collective housing; domesticity; rationalization; mechanization; kitchen; domestic engineers

Persona de contacto / Corresponding author: carmen.espegel@upm.es. Escuela Técnica Superior de Arquitectura, Universidad Politécnica de Madrid. España 
1. Portadas de los Manuales: The American Woman's Home, 1869; The New Housekeeping, 1913; The Psychology of Management, 1914, y los retratos de sus autoras: Catharine Beecher, Christine Frederick y Lillian Gilbreth.
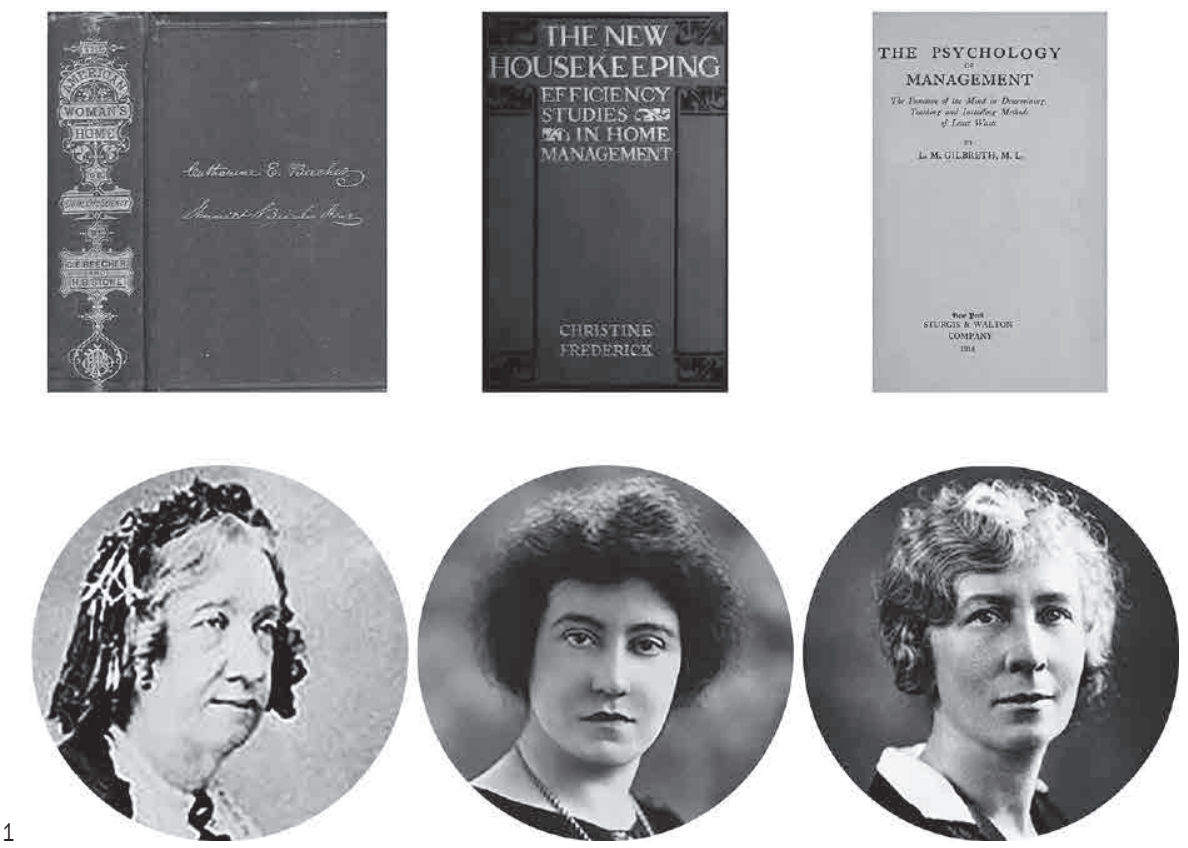

C on los avances técnicos surgidos en los inicios del siglo veinte, los principales arquitectos europeos: Gropius ${ }^{1}$, Behne $^{2}$, Le Corbusier ${ }^{3}$, Ginzburg ${ }^{4}$ o incluso Mendelsohn ${ }^{5}$, observaban con admiración los Estados Unidos en términos de producción industrial en serie y métodos de eficiencia en el trabajo. Todos veían plasmadas sus inquietudes teóricas en las propuestas de Taylor y Ford, por lo cual intentaron transferir esos logros hacia la arquitectura moderna, al ser en cierto modo componentes fundamentales de una renovación socialal.

Al mismo tiempo, la élite de la intelligentsia europea de signo izquierdista, Bruno Taut o Ernst May, cuando ideó la vivienda para la clase trabajadora, también miraba hacia América, pero hacia unas investigaciones realizadas por no profesionales. Los Manuales escritos por Catharine Beecher, Christine Frederick y Lillian Gilbreth (figura 1) tuvieron una enorme influencia sobre la concepción de la domesticidad en los programas residenciales de la Nueva Oficina de Construcción de Frankfurt, liderada por Ernst May ${ }^{7}$ entre 1925 y 1930, a través de Margarete Schütte-Lihotzky ${ }^{8}$.

Descubriremos los insólitos caminos de transmisión de esas exploraciones estadounidenses al contexto europeo, fuera de los habituales circuitos de las teorías arquitectónicas. Pues los técnicos alemanes, con un entendimiento social de su trabajo y una mirada abierta hacia la participación ciudadana, dialogaron con las asociaciones de mujeres, quienes finalmente introdujeron el progreso americano en la modernización de

1. GROPIUS, Walter. Die Kunst in Industrie und Handel. Jena: Verlegt bei Eugen Diederichs, 1913.

2. BEHNE, Adolf. Der Moderne Zweckbau. Vienna/Berlin: Drei Masken Verlag, 1923.

3. LE CORBUSIER-SAUGNIER. Vers une architecture. Paris: Les Editions G. Cres et Cie., 1923.

4. GINZBURG, Moisei. Stil'i epokha. Moskva: Gosizdat, 1924.

5. MENDELSOHN, Erich. Amerika: Bilderbuch eines Architekten. Berlin: Rudolf Mosse Buchverlag, 1926.

6. MCLEOD, Mary. Architecture or Revolution: Taylorism, Technocracy, and Social Change. En: Art Journal, vol. 43, n. ${ }^{\circ}$ 2, pp. $132-147$.

7. Ernst May und das neue Frankfurt 1925-1930, Catálogo de la Exposición en el Deutschen Architekturmuseums, Frankfurt am Main. Berlin: Ernst \& Sohn, 1986.

8. NOEVER, Peter (ed.). Margarete Schütte-Lihotzky. Soziale Architektur Zeitzeugin eines Jahrhunderts. Wien: Bóhlau, 1993. 
N18_ARQUITECTURAS AL MARGEN

60

Imágenes extraídas del libro de Catharine Beecher y Harriet Beecher Stowe, The American Woman's Home. 3. Sótano, planta baja y planta primera del proyecto de vivienda mostrado en el Manual de Catharine Beecher y Harriet Beecher Stowe, The American Woman's Home.
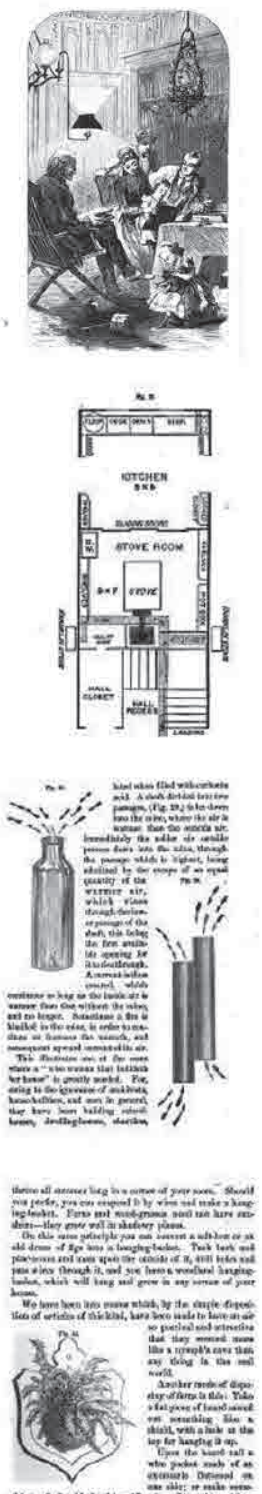

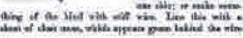
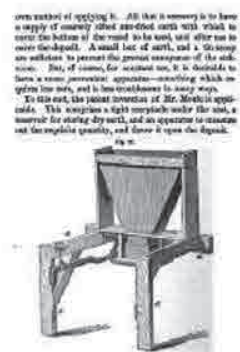
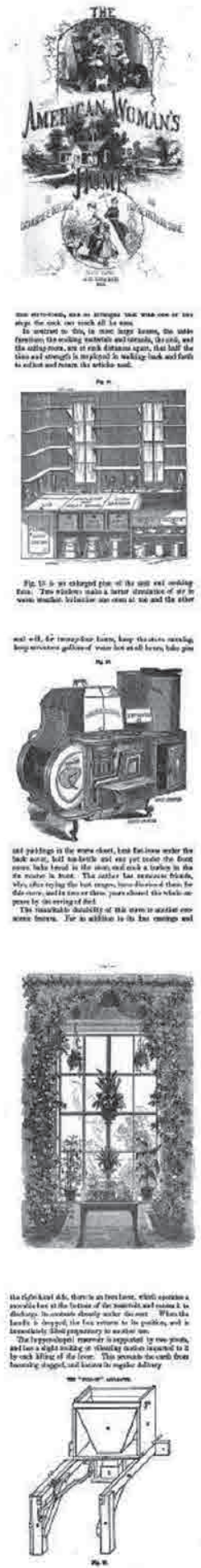
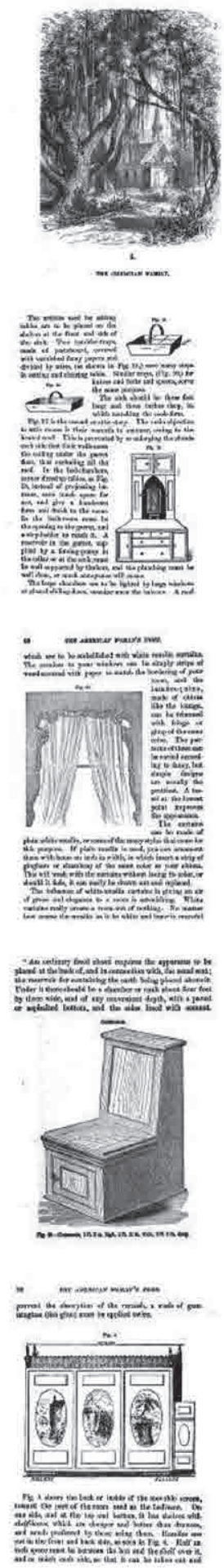
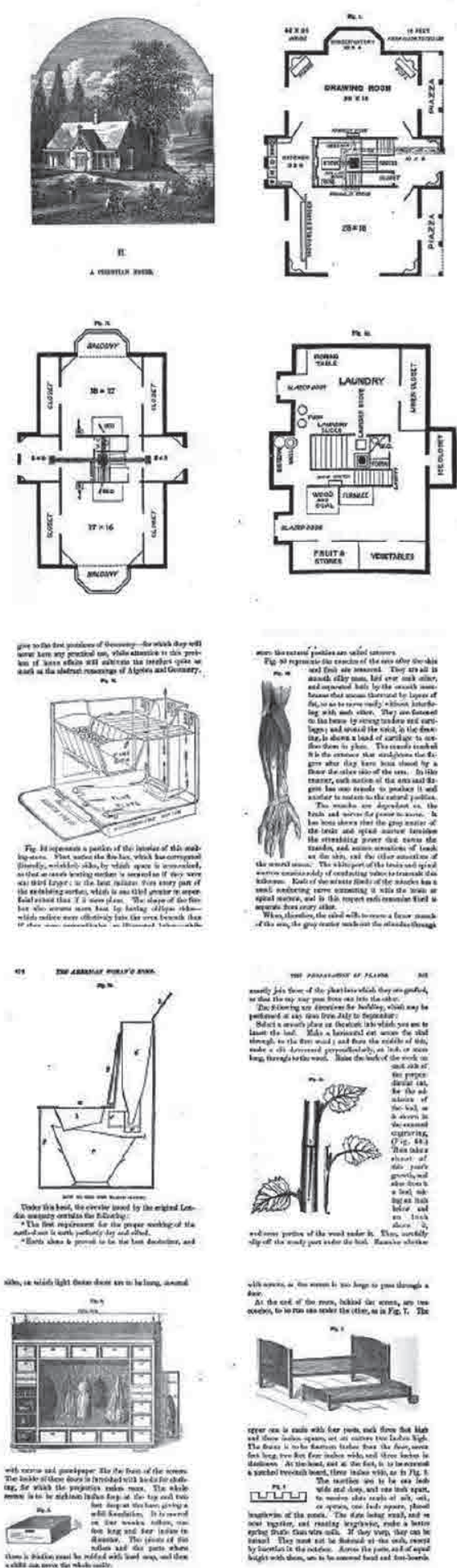

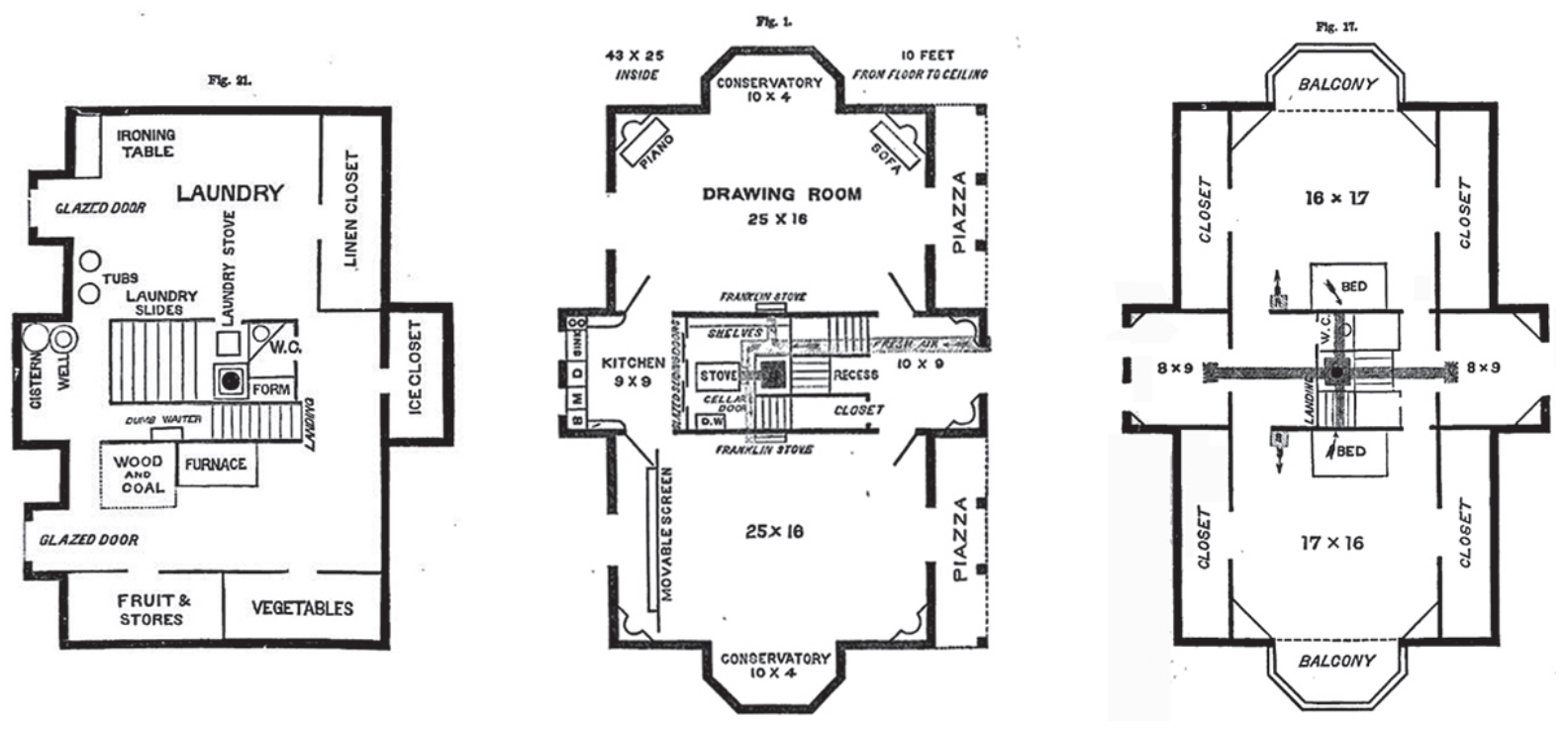

la vivienda de entreguerras. Como veremos, lo que en principio fueron unos compendios enfocados hacia el mundo femenino llegaron a ser el leitmotiv de los pioneros de la vivienda social.

\section{PERFILES. UN ENFOQUE INTELECTUAL, PRAGMÁTICO Y CIENTÍFICO.}

Catharine Beecher (1800-1878) fundó el Seminario Femenino Hartford en 1823, una escuela para jóvenes damas con la que sobrepasó los límites de la educación tradicional al establecer un plan de estudios que incluso englobaba las ciencias teóricas y la actividad física. Su espíritu racional, aunque muy sensible al observar la realidad, le indujo a escribir varios libros. Entre ellos destaca A Treatise on Domestic Economy, for the Use of Young Ladies at Home and at the Schoo/ ${ }^{9}$, considerada la primera guía completa para el mantenimiento del hogar que se publica en Estados Unidos.

El siguiente libro, The American Woman's Home or Principles of Domestic Science Being a Guide to the
Formation and Maintenance of Economical Healthful Beautiful and Christian Homes ${ }^{10}$, lo firma con su hermana Harriet Beecher Stowe ${ }^{11}$ (figura 2). Este documento es una descripción rigurosa de todas las tareas del hogar, paso a paso, explicadas de forma precisa y donde la mecanización será el término clave del ama de casa "profesional". En la portada descubrimos su mundo ideal, que consiste en el hogar, la familia y la iglesia, pero un hogar ilustrado, donde todos sus miembros leen y lo hacen bajo una luz diseñada para ser eficiente, pues mientras brilla tenue hacia el techo, ilumina con intensidad el área de lectura. Un libro para afrontar la vida y su organización, con la familia en el núcleo.

Su eficaz transformación del hogar se centra en una columna vertebral (figura 3), un elemento técnico que contiene no sólo escaleras, armarios, inodoro, cocina y fogón sino el sistema de conductos de calefacción que impulsan el aire caliente desde una caldera en el sótano hasta las estancias, así como los de ventilación con rejiIlas de entrada y salida para renovar el aire ${ }^{12}$. También hay

9. BEECHER, Catharine. A Treatise on Domestic Economy, for the Use of Young Ladies at Home and at the School. Boston: Marsh, Capen, Lyon and Webb, 1841. 10. BEECHER, Catharine y BEECHER STOWE, Harriet. The American Woman's Home or Principles of Domestic Science Being a Guide to the Formation and Maintenance of Economical Healthful Beautiful and Christian Homes. New York: J. B. Ford and Company, 1869.

11. Conocida por haber escrito La cabaña del tío Tom o La vida entre los humildes, en 1852.

12. Catharine Beecher tuvo una salud delicada y siempre se preocupó por la correcta ventilación de las viviendas. Tal es el motivo por el cual enfatizó el diseño de una aireación dinámica para cada estancia. Como ejemplo de esta mecanización progresiva de la casa, el libro también está repleto de patentes sobre soluciones innovadoras. Su método implicó la búsqueda de una eficiencia que garantizase la máxima salubridad. 

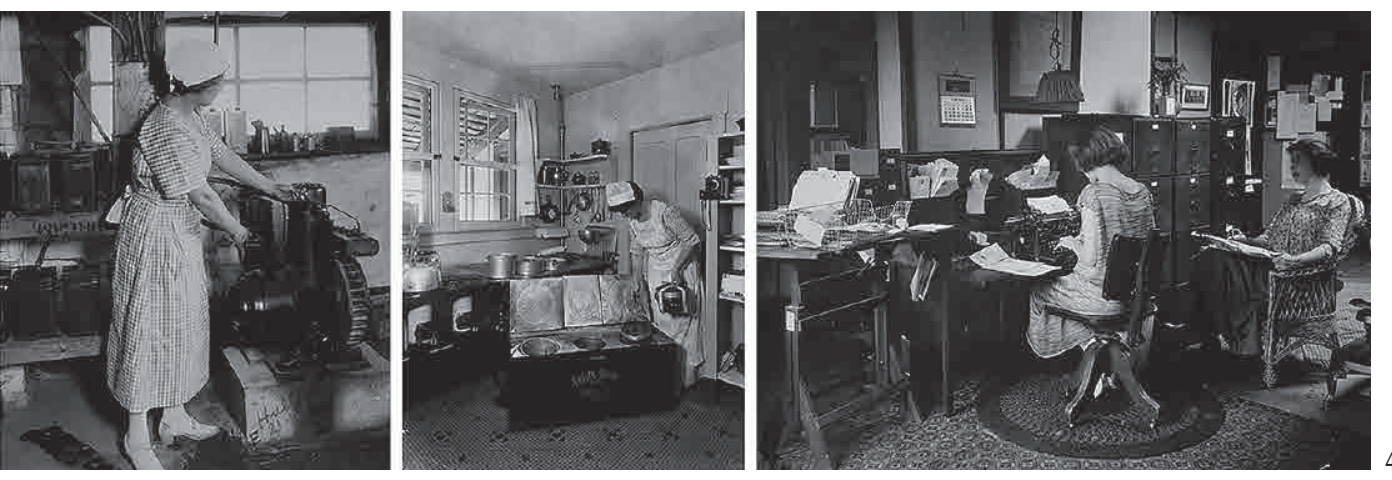

4. En estas tres fotografías observamos a Christine Frederick en lo que fue su laboratorio, el Applecroft Home Experiment Station, mientras realiza diferentes tareas domésticas: engrasar maquinaria, utilizar una caja de heno que servía de cocina económica sin fuego o recabando notas en su oficina instalada en el ático.

5. Diagramas de organización del mobiliario en la cocina publicados por Christine Frederick en The New Housekeeping

mejoras de tipo espacial con cuartos transformables mediante armarios móviles y paneles deslizantes, dirigidos a reducir el tamaño de la casa, además de por razones económicas, para un uso, cuidado y mantenimiento más eficaz.

Sin embargo, existe una diferencia sustancial con los proyectos precedentes de Beecher: la consciente "no definición" de los usos de las habitaciones. Son los muebles de su interior, el piano o la cama, los que cualifican el uso preciso de esos cuartos. La flexibilidad se maximiza con muebles-pantallas decorativos que ocultan el vestidor o las camas supletorias. Por otro lado, con una encimera única de altura continua, la cocina se ha convertido en un espacio de proceso racional, donde se descubren dos áreas separadas: una para guisar al fuego y otra para la preparación de la comida.

En realidad, Beecher no escribió simples manuales o breviarios de cocina, sino exhaustivos tratados, libros de texto integrales con un espíritu muy próximo al de la ilustración del siglo XVIII'13, una forma de conocimiento razonado, una primera explicación sistemática de la economía doméstica, todo en aras de fomentar la buena salud familiar

Para conocer a la siguiente protagonista, Christine Frederick (1883-1970) basta con utilizar sus propias palabras en Selling Mrs. Consumer ${ }^{14}$ al describir su amplio perfil profesional: consejera de marketing para los fabricantes de artículos para el hogar; fundadora de la primera organización sobre publicidad femenina; primera en aplicar los principios de gestión científica al hogar. Se graduó en la Universidad de Northwestern y se dedicó a la enseñanza. Más tarde, gracias a los socios de su marido conoció de primera mano el valor de la organización científica del trabajo. Con ayuda de estos colegas, trató de aplicar esos principios en el hogar, con todos sus problemas implícitos, ya que en la fábrica se realizan pocos procesos y se repiten en bucle mientras que en el hogar las tareas son infinitas y se superponen: un problema de escala. En 1912, comenzó a explicárselo a las amas de casa de clase media, gracias a las páginas del Ladies' Home Journal.

Sus textos abogaban por una mayor eficiencia en la cocina con el argumento de que las amas de casa debían disfrutar de un equipo idóneo y experimentar con técnicas renovadas, al igual que hacían los empresarios y agricultores. Para defender sus teorías, Frederick organizó una cocina modelo, el Applecroft Home Experiment Station, en su propio hogar de Long Island (figura 4). Con ello, su casa se convirtió en un laboratorio donde ella actuaba de investigadora y de objeto de estudio simultáneamente. Este experimento generó una larga indagación práctica que se prolongó desde 1910 hasta 1930.

El libro The New Housekeeping: Efficiency Studies in Home Management ${ }^{15}$ es un resumen de la obra que publicó por entregas en The Ladies Home Journal. La gestión científica significaba eficiencia y ésta se conseguía

13. Incluso los dibujos nos remiten al grafismo enciclopedista de Diderot y su taxonomía ilustrada donde todo se articula y se desmonta para lograr un conocimiento libre de prejuicios adquiridos.

14. FREDERICK, Christine. Selling Mrs. Consumer. New York: The Business Bourse, 1929.

15. FREDERICK, Christine. The New Housekeeping: Efficiency Studies in Home Management. Garden City (New York): Doubleday, Page \& Company, 1913. 


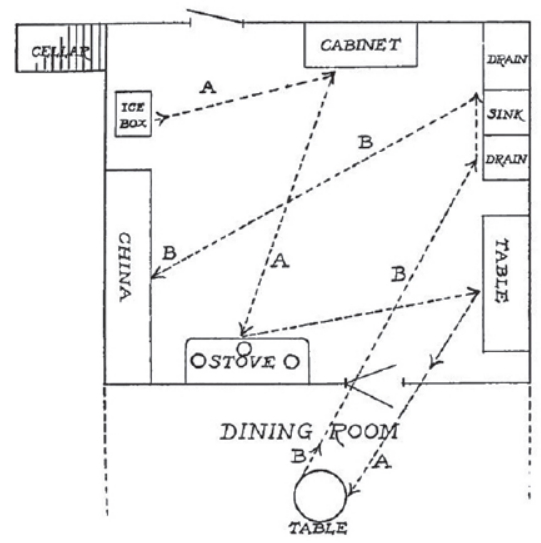

Diagram showing badly arranged equipment, which makes confused intersecting chains of steps, in either preparing or clearing away a m
(A-preparing; $B$ - clearing)

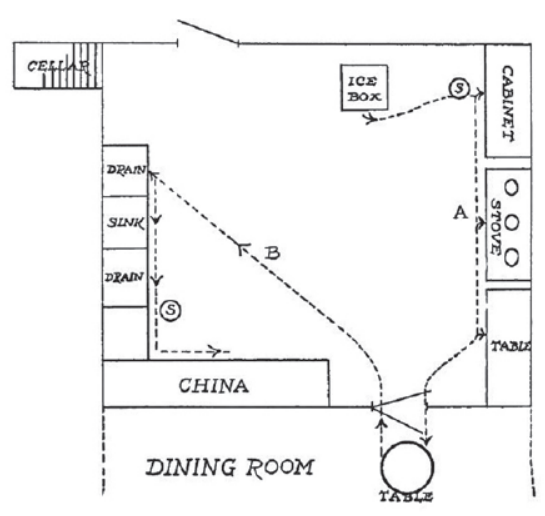

Diagram showing proper arrangement of equipment, which makes a simple chain of steps, in either prepar-

ing or clearing a way a meal.
(A - preparing; i - clearing mediante la introducción de los novedosos electrodomésticos, ya más asequibles. En este manual encontramos los conocidos diagramas que muestran una incorrecta organización de la cocina y otra apropiada, donde la diferencia radica en la ubicación de los muebles y los aparatos, que distingue dos circuitos: uno limpio con la salida de los alimentos preparados hacia el comedor y otro sucio con la entrada de la vajilla para lavar (figura 5).

En el siguiente compendio, Household Engineering: Scientific Management in the Home ${ }^{16}$, realizó una descripción específica de las labores domésticas, que enumera, analiza, reflexiona y programa, para finalmente redefinirlas de un modo más eficiente (figura 6). Su alegato en favor de los utensilios domésticos o de los equipos mecánicos se comprende desde la óptica de la casa sin personal de servicio. Dignificó el trabajo del hogar con el simple hecho de elegir el utensilio adecuado para cada tarea, ya que, al precisar los métodos de limpieza, entendió que si se empleaba el instrumento correcto la labor podría ser más fácil y menos pesada para el ama de casa.

Destaca en su obra el pragmatismo que inundaba todos los análisis, que condujeron a introducir la estandarización en la altura de las encimeras y al fomento del correcto diseño de cocinas para evitar recorridos innecesarios. En sus textos abrazó la publicidad como una manera de informar a las mujeres sobre los beneficios de la nueva era. Al extender los principios científicos al hogar, su trabajo también llevó la esfera doméstica a un sistema industrial que estaba reconfigurando los negocios y la política. La mayor transformación propuesta por ella fue un cambio de percepción para integrar la vida de las organizadoras del hogar en el nuevo orden económico y social.

Los terceros protagonistas, los Gilbreth, Lillian (18781972) y Frank (1868-1924), serán conocidos por ser pioneros en la administración o gestión científica, con lo que denominaron Time-Motion Studies, empleando la grabación cinemática para estudiar los procesos y la velocidad de producción. En 1914, Lillian publica The Psychology of Management: The Function of the Mind in Determining, Teaching and Installing Methods of Least Waste ${ }^{17}$, donde se diserta sobre el bienestar laboral, los incentivos y la prevención de accidentes. La supresión gradual del cansancio gratuito, bien físico o psicológico, le llevará a redactar en 1916, Fatigue Study: The Elimination of Humanity's Greatest Unnecessary Waste; A First Step to Motion Study ${ }^{18}$

La clave de su método consistía en pautar el espacio, medir el tiempo y filmar los procesos de trabajo para después analizarlos y optimizarlos (figura 7). Motivados por las técnicas de los Time Study Work de Taylor y su implantación de tiempos estándar, los Gilbreth, en su Motion Study Work, propusieron un nuevo lenguaje técnico, lo que facilitó un análisis del procedimiento operativo bajo una óptica científica. El matrimonio utilizó su bagaje técnico previo para desarrollar un método basado en el análisis pormenorizado de los movimientos, que en parte consistía en una filmación

16. FREDERICK, Christine. Household Engineering: Scientific Management in the Home. Chicago: American School of Home Economics, 1920.

17. GILBRETH, Lillian. The Psychology of Management: The Function of the Mind in Determining, Teaching and Installing Methods of Least Waste. New York: Sturgis \& Walton Company, 1914.

18. GILBRETH, Frank B. y GILBRETH, Lillian. Fatigue Study: The Elimination of Humanity's Greatest Unnecessary Waste. A First step in Motion Study. New York: Sturgis \& Walton Company, 1916. 
N18_ARQUUTECTURAS AL MARGEN

64

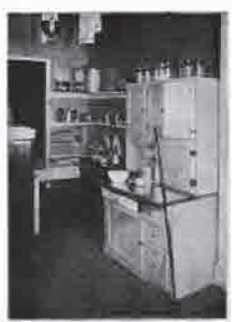

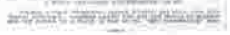
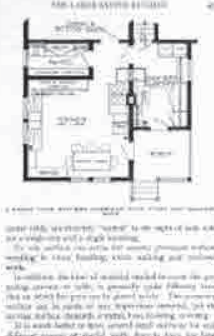$$
\text { C. T. T T }
$$$$
\frac{1}{-1}-1
$$

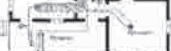$$
\text { TIT T-T }
$$$$
+T
$$$$
1-1,1-7
$$
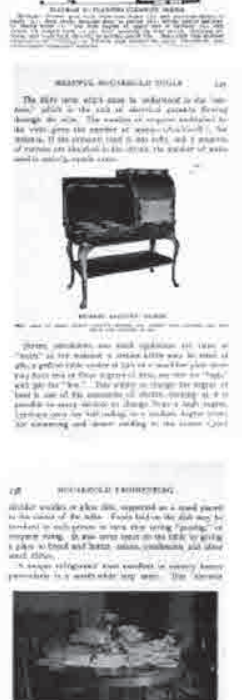
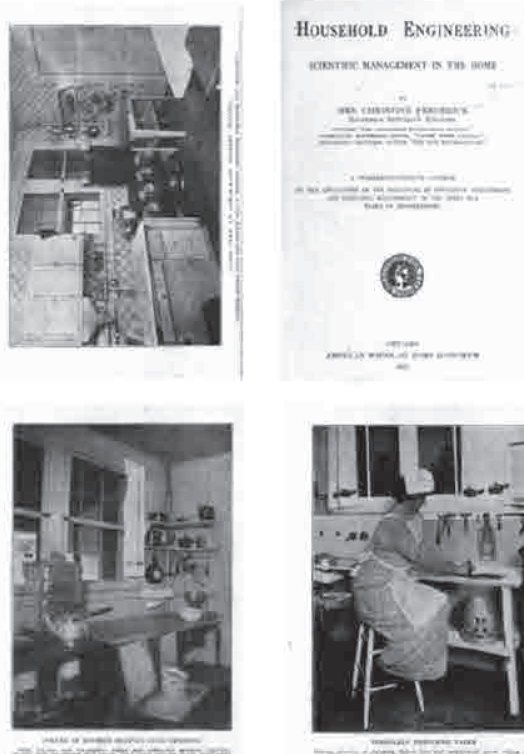

(6)
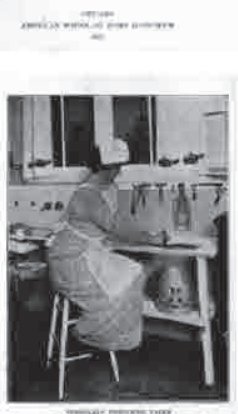

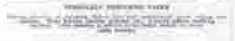
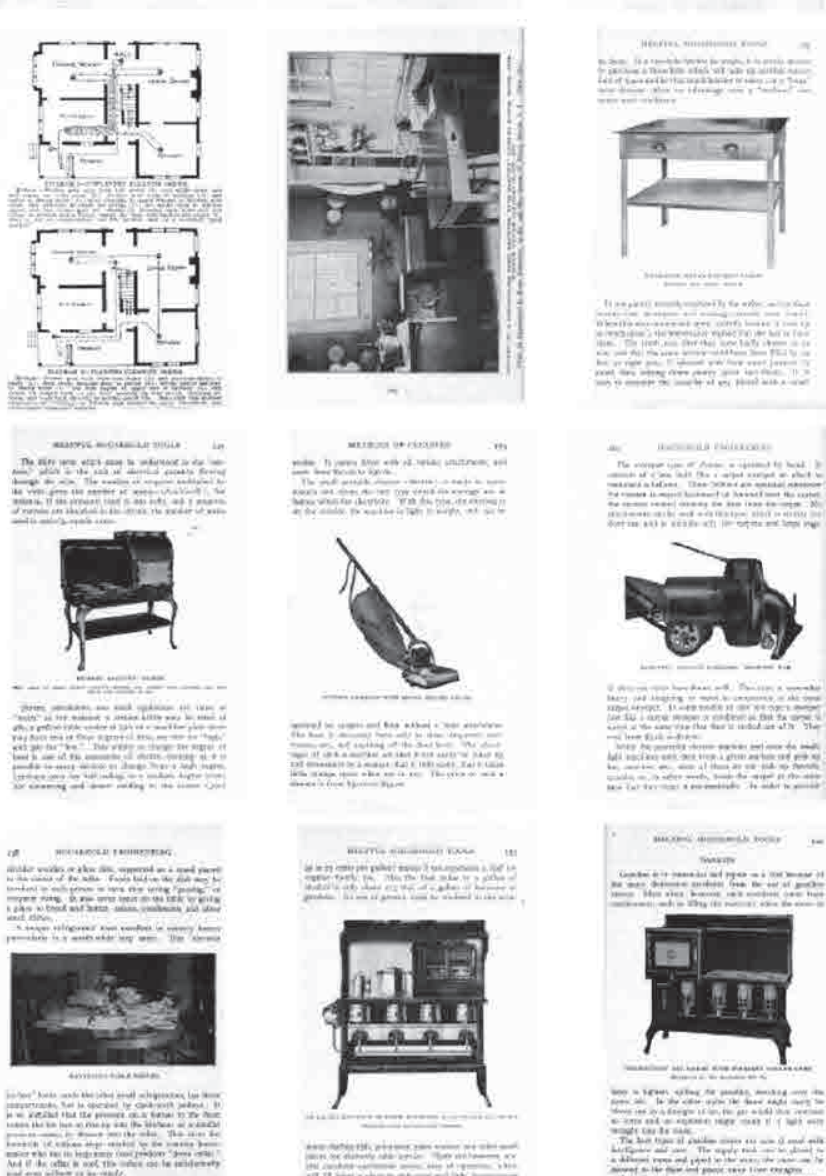

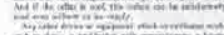
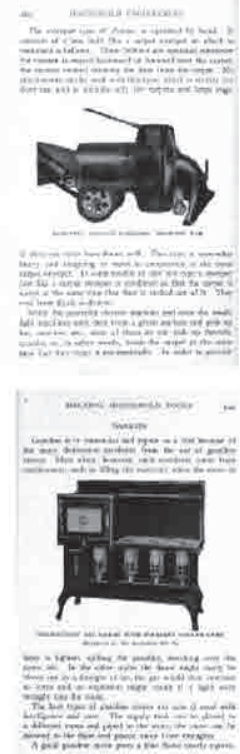

6. Imágenes del libro de Christine Frederick, Household Engineering.

7. Micro-cronómetro para estudio de micro-movimientos, circa 1915

8. Cronociclógrafo recogiendo los movimientos de una operaria, circa 1914.
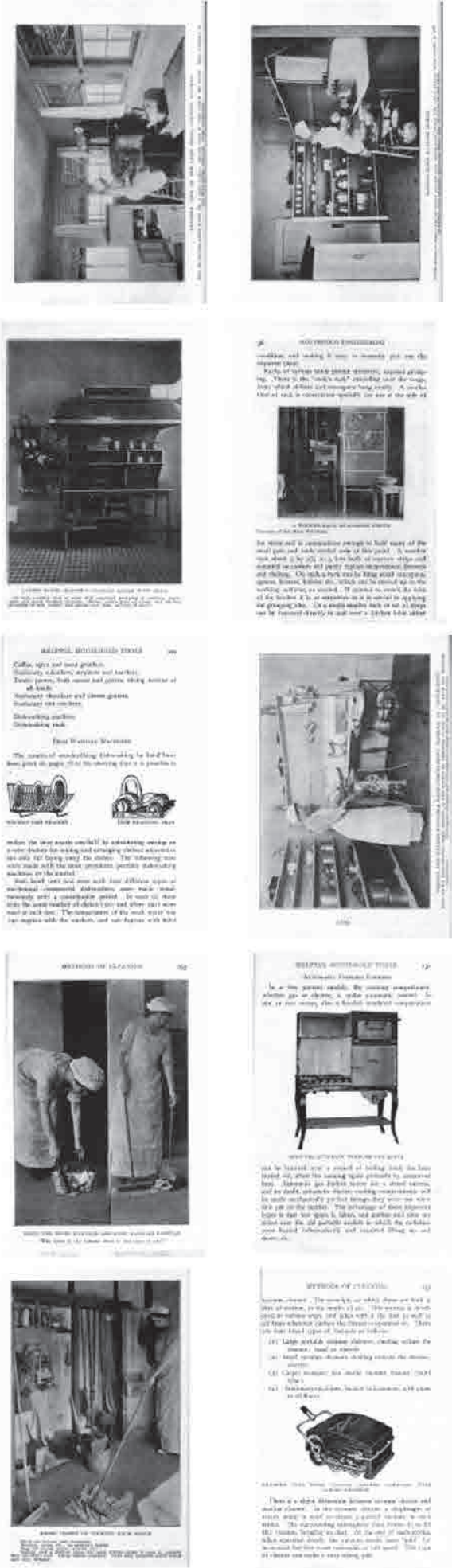


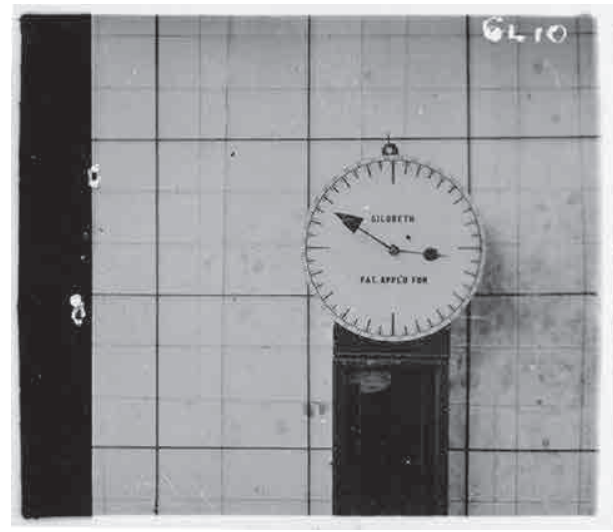

7
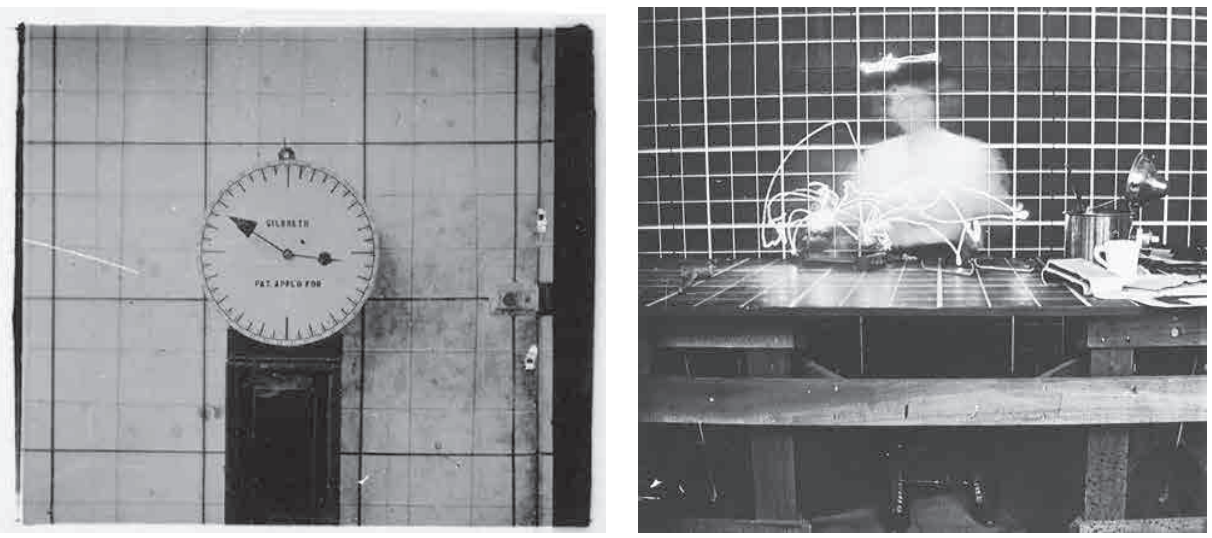

8 detallada de las acciones de un trabajador y de su postura corporal mientras se grababa. Sus películas ${ }^{19}$ muestran múltiples operaciones industriales a partir de las cuales se desarrolló la técnica de estudio de movimientos.

Su interés en un análisis racionalizado del tiempo implicó el poder visualizarlo adecuadamente, mediante unos diagramas dinámicos. Así, en el "Ciclógrafo de una experta dobladora de pañuelos" (figura 8), los Gilbreth sujetaron una bombilla en el dorso de la mano operaria y utilizaron un tiempo de exposición lento para fotografiar el trazado del movimiento como si fuera una línea de luz continua en el espacio.

Tras la muerte de Frank en 1924, Lillian continuó su legado, aunque se centró en su especialidad, la psicología de la gestión ${ }^{20}$. Destacó la importancia del "factor humano", precursor de la ergonomía, en la aplicación de las técnicas científicas y demostró a los gerentes que la producción se incrementaba con una mejor iluminación o con períodos de descanso. Además, encaminó estas observaciones hacia el hogar, con una mejora de los electrodomésticos, el diseño de "la cocina eficiente" y después la primera cocina con todas las facilidades para personas discapacitadas o delicadas del corazón

\section{REFERENCIAS. CRÓNICA DE UNA TRAVESÍA DE IDEAS.}

Como se ha citado, los grandes arquitectos europeos admiraban Norteamérica por su producción industrial y sus métodos de eficiencia fabril. Taylor, con su estudio del tiempo en el trabajo, así como Ford y su cadena de montaje, nos remiten a los conceptos de eficacia, perfección, economía, productividad y precisión. Estas ideas inspiraron a los europeos que querían transferir sus logros industriales hacia la arquitectura moderna. Imágenes de industrias, silos agrarios o líneas de montaje eran las concreciones de un sistema de mercado, el capitalismo americano, que primaba la producción en masa.

En cambio, para las referencias utilizadas en el hábitat de entreguerras, Europa asimila el trabajo de estas pioneras. Bruno Taut publicó en Berlín Die Neue Wohnung: Die Frau als Schöpferin ${ }^{21}$, en 1924. La nueva vivienda sería su traducción, pero el subtítulo menciona a La mujer como diseñadora, literalmente, "creadora". Al comienzo del libro, se cita a Frederick y su manual The new housekeeping, con los diagramas circulatorios para una mejor organización del espacio habitable.

Podría pensarse que tal vez fuera Bruno Taut el que introdujo a Margarete Schütte-Lihotzky en los trabajos publicados por Christine Frederick, ya que mantuvieron

19. Rodadas entre 1910 y 1924, fueron patrocinadas por la Sociedad para el Progreso de la Gestión de Chicago.

20. Lillian Gilbreth estudió literatura inglesa en la Universidad de California, Berkeley, graduándose en 1900. Después se decidió por un Master en Psicología en la Universidad de Columbia en Nueva York, aunque realizó su doctorado en Berkeley durante 1911, en Psicología de la Gestión, donde no logró el título por prejuicios de género. Esto le obligó, en 1915, a obtener otro doctorado en la Universidad de Brown en Providence, Rhode Island, bajo la premisa Some Aspects of Eliminating Waste in Teaching.

21. TAUT, Bruno. Die Neue Wohnung. Die Frau als Schöpferin. Leipzig: Klinkhardt \& Biermann, 1924, p. 14 y pp. 65-66. 
9. Transferencias entre Estados Unidos, Europa y Japón a través de las traducciones de libros, conferencias, congresos y exposiciones de Harriet Beecher Stowe, Christine Frederick y Lillian Gilbreth.

10. Mapa que recoge la denominación y ubicación en Centroeuropa de algunas asociaciones femeninas en la época de la República de Weimar.

una profunda amistad. Pero también barajamos una versión más plausible que implica a Erna Meyer ${ }^{22}$, asesora de J.J.P. Oud en el diseño de la cocina para las viviendas de la Weissenhof (1927) ${ }^{23}$, y a Irene Witte, esta última fiel traductora al alemán de los estudios de Frederick y los Gilbreth. Ambas, vinculadas a la red de asociaciones femeninas y que ya manejaban las avanzadas ideas de las americanas, bien pudieron enseñar a Lihotzky la corriente estadounidense sobre la eficacia en la vivienda. La divulgación de las transferencias que hubo desde América a Europa se produjo por diversos medios: la traducción de los manuales, los artículos en revistas femeninas, las conferencias en territorio europeo, así como la difusión de su trabajo por medio de las asociaciones de mujeres, en auge durante esos años (figura 9).

Como pionera y para promover la lucha contra la esclavitud, Harriet Beecher Stowe viajó a Inglaterra en 1853, aunque después realizó dos giras, a París y a Roma. Durante estos viajes, daría a conocer no sólo su trabajo sino el Tratado de Economía Doméstica de su hermana Catharine. Si bien, la figura más importante en cuanto a diseminación de sus ideas fue Christine Frederick cuyos trabajos serían publicados en las principales capitales europeas $^{24}$. Además, durante los años veinte, impartió numerosas conferencias ${ }^{25}$ sobre el avance científico en los Estados Unidos, trabajando en estrecha cooperación con las amas de casa. Por otro lado, Lillian Gilbreth intervino en múltiples congresos ${ }^{26}$ presentando sus estudios sobre el movimiento. Su amiga Irene Witte, estudiosa de
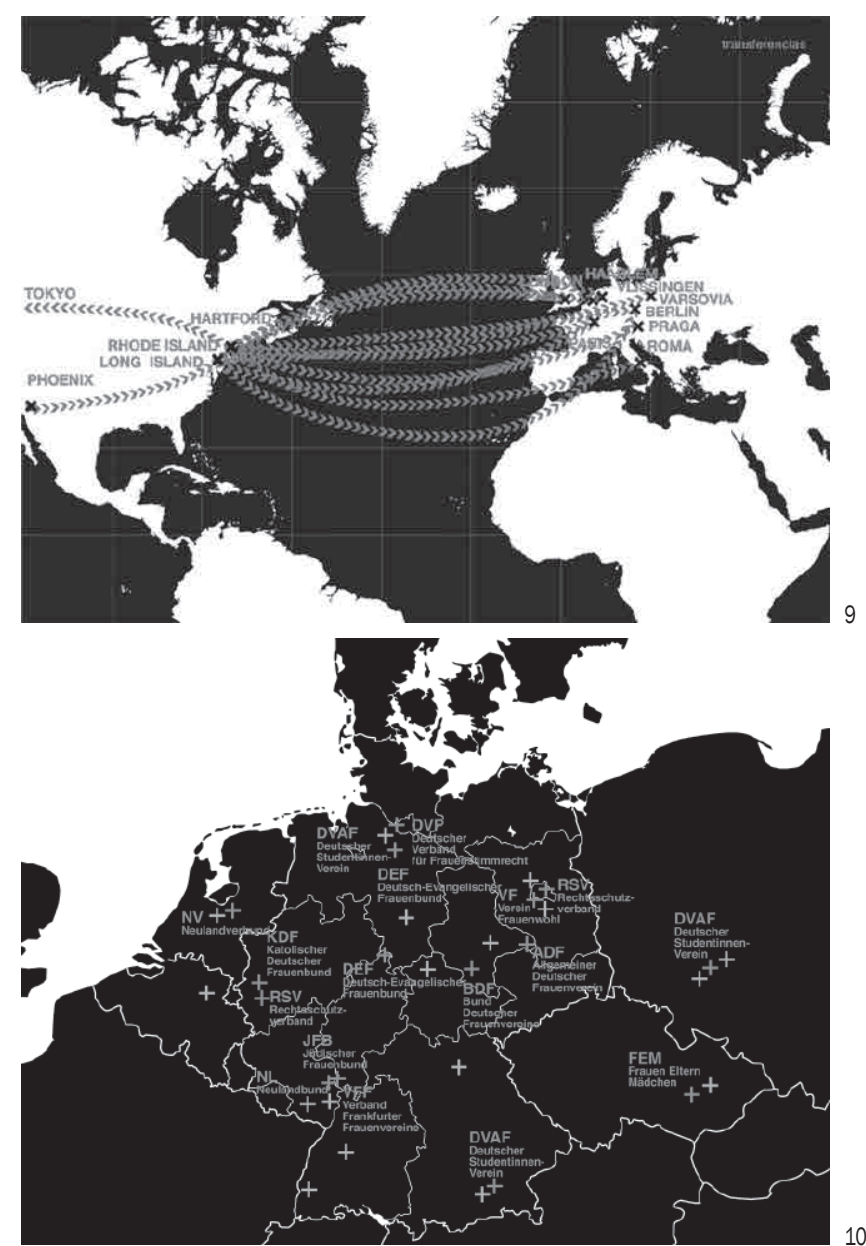

22. MEYER, Erna. Der neue Haushalt ein Wegweiser zu wirtschaftlicher Haushaltsführung. Stuttgart: Franckh'sche Verlagshandlung, 1926.

23. B00T, Marjan y CASCIATO, Maristella (ed.) La casalinga riflessiva. La cucina razionale come mito domestico negli anni '20 e '30. Catálogo de la Exposición abierta entre octubre y noviembre de 1983 en el romano Palazzo delle Esposizioni. Roma: Multigrafica Editrice, 1983.

24. Bajo el título Le Taylorisme chez soi. Pratique de la direction de la maison (Paris: Dunod Editeur, 1920) fue publicado, en Francia, el primer manual de Frederick. Al año siguiente, se editó en alemán con el título Die rationelle Haushaltführung. Betriebswissenschaftliche Studien (Berlin: Julius Springer Verlag, 1921), traducido por Irene Witte. También fue traducido al polaco con el título Naukowa organizacja w gospodarstwie domowen (Warsaw: Nakladem Instytuta Naukowej Organizacji, 1926), con prólogo y epílogo de Henry Le Chatelier, un conocido científico que fomentó el desarrollo industrial. La versión holandesa, publicada con el título De Denkende Huisvrouw, Nieuwe Inzichten (El ama de casa pensante, nuevas ideas) (Haarlem: Tjeenk Willink, 1928), contiene una introducción escrita por E.J. van Waveren-Resink, activista en la Asociación Holandesa de Amas de Casa. Por último, la traducción italiana realizada por Lorenzo Tealdy llevaba el título La donna e la casa. II taylorismo della vita domestica (Torino: C. Accame, 1928).

25. Tenemos constancia de sus disertaciones en siete países europeos: Inglaterra, Francia, Bélgica, Holanda, Italia, Alemania y Suiza.

26. En Berlín impartió nueve conferencias en 1920, traducidas por Irene Witte. Asimismo, en 1924, participó en el Primer Curso en Europa sobre Motion Study impartido en Praga y en el Primer Congreso Internacional de Gestión. Mientras que en 1925, intervino en el IRI Congress en Vlissingen (Holanda), donde aportó la mayor parte del léxico científico para la materia. 
11. Izquierda: La vivienda definida por Catharine Beecher y Harriet Beecher Stowe en The American Woman's Home. Derecha: Siedlung Römerstad 1927-1928. Das Neue Frankfurt.
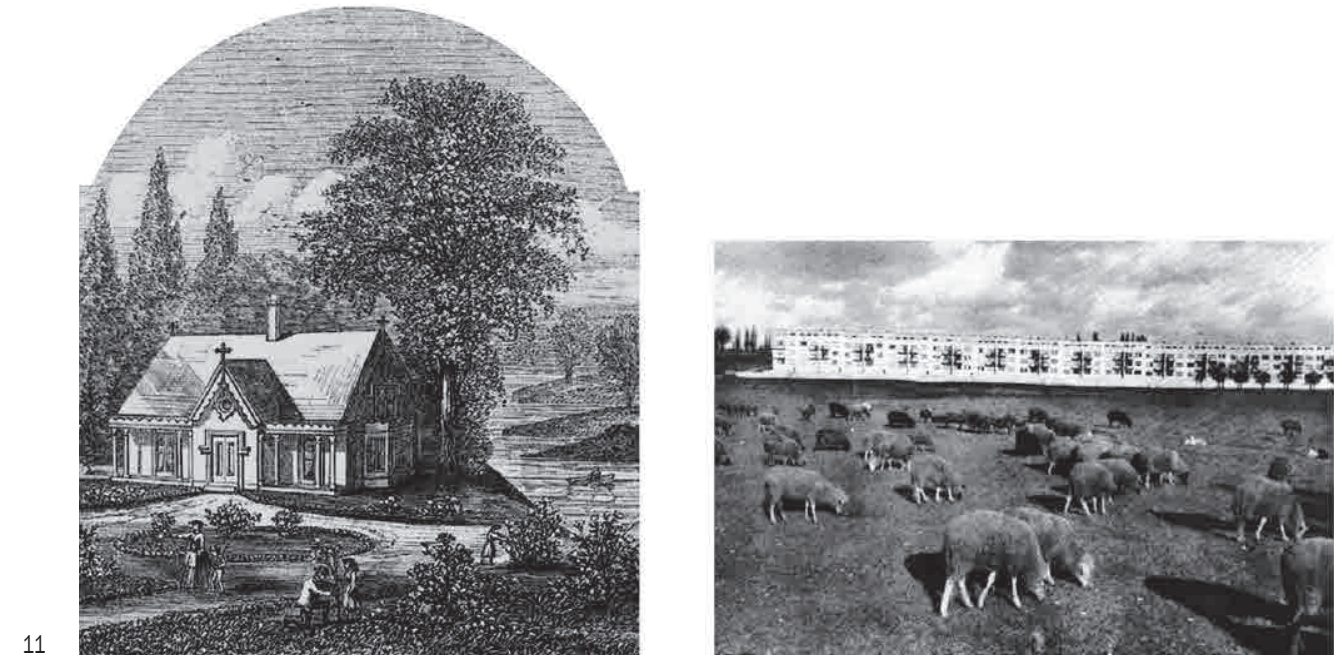

Taylor y los métodos de eficacia en el trabajo, tradujo al alemán todos sus artículos y libros ${ }^{27}$.

Entre las revistas de la época, sobresale la publicada por Paulette Bernège entre 1923 y 1930, Mon chez moi. La revue d'organisation ménagère, donde la editora escribió entre otros el artículo "Vers une cuisine automatique" 28 , en clara alusión al famoso libro de Le Corbusier. Trata sobre los aparatos utilizados en una cocina americana, con unos diagramas similares a los de Frederick, con el trazado de pasos y distancias, incluso con planos y esquemas originales de la americana.

Al terminar la Gran Guerra, gracias a la aprobación del sufragio universal por la República de Weimar, se produce un aumento exponencial de las asociaciones femeninas (figura 10): el nuevo valor del voto las hace visibles y decisivas. También en el resto de Europa -Polonia, Austria, Países Bajos y Bélgica- proliferaron estas federaciones, ya fueran religiosas de signo conservador, feministas, estudiantiles, de tipo político, laborales o simplemente de amas de casa. Estas agrupaciones fomentaron la influencia social, la labor de enseñanza o una defensa de los derechos de las mujeres. Gracias a estas redes se canalizó el conocimiento de las incipientes ideas norteamericanas y su aplicación práctica en el hogar.

\section{TRANSFERENCIAS. LAS REPERCUSIONES CÍVICA Y CULTURAL.}

Ahora, analizaremos algunos aspectos del modelo americano y sus transferencias al entorno alemán. Empezaremos con la concepción urbana estadounidense y sus viviendas vinculadas al campo frente a las Siedlungen más cercanas al concepto inglés de ciudad jardín (figura 11). A finales del siglo XVIII, Thomas Jefferson trató de representar un esquema del espacio ideal democrático, con el fin de primar la granja familiar sobre el crecimiento de las ciudades en Nueva Inglaterra. En ese momento, se debatía sobre el modelo urbano idóneo para toda una nación y la utopía agraria de la que surge la visión de Jefferson no era ecuánime, ya que favorecía el predominio de un estamento social: el Sur más tradicionalista. En general, las familias burguesas abandonaron el centro de la ciudad para instalarse en viviendas suburbanas. Los primeros prototipos de la periferia diseñados por Catharine Beecher, fueron imaginados como espacios para el trabajo doméstico femenino al servicio de la familia.

En cambio, Ernst May propuso un crecimiento polinuclear de Frankfurt, por medio de diversos satélites que funcionaban como unas colonias autosuficientes, casi autárquicas. Los asentamientos debían establecerse cerca de la extensa naturaleza y separados del núcleo

27. Frank B. y Lillian M. Gilbreth: Ermüdungsstudium (Fatigue Study). Eine Einführung in das Gebiet des Bewegunǵsstudiums. Verlag des Vereines Deutscher Ingenieure. Berlin, 1921. Lillian Gilbreth: Verwaltungsspsychologie. Berlin: Verl. d. Vereins dt. Ingenieure, 1922.

28. BERNĖGE, Paulette. Vers une cuisine automatique. En: Mon Chez Moi. Fascículo IV, 1 de Octubre 1923 - 1 de Noviembre 1923, pp. $25-29$. 

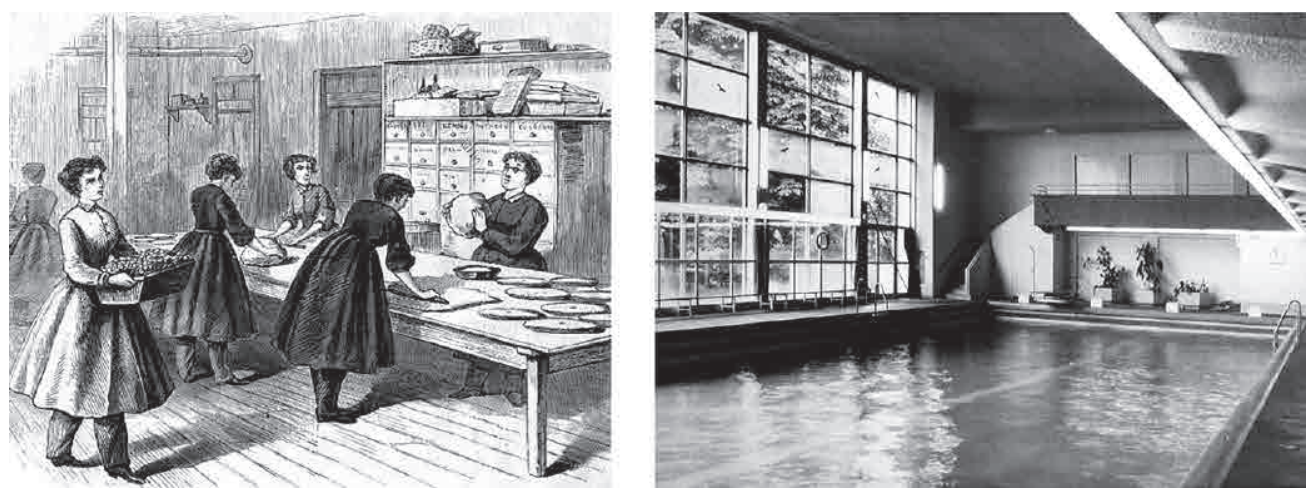

12. Izquierda: Panadería comunitaria norteamericana. Derecha: Piscina cubierta pública en Frankfurt am Main, del arquitecto Martin Elsaesser, 1929.

13. Superior izquierda: restitución de la planta de la vivienda de las Beecher, con el mueble-pantalla móvil, y detalles de cama supletoria y del mueble-pantalla por ambos lados. Superior derecha: restitución de la planta de la casa mínima tipo "Zwofa" (2 familias) con mobiliario, 1928, Frankfurt am Main. Inferior: fotografías del estar diurno y mobiliario nocturno en la Siedlung Praunheim, 1926-1929.

14. Izquierda: Christine Frederick en el Applecroft Home Experiment Station. Derecha: la cocina Frankfurt, 1926. tradicional urbano por un anillo verde. En cuanto a la disposición general se basaba en la repetición de filas de las viviendas, orientadas casi de manera estricta Este-Oeste, aunque con ciertas variaciones para adaptarse a la topografía o a la red viaria. Las 15.000 unidades levantadas bajo la dirección de May representaron más del noventa por ciento de las viviendas terminadas en esa ciudad en ese período. Esta impresionante cifra difícilmente podría lograrse sin el empeño que se puso sobre la eficiencia económica desde el diseño a la construcción.

El modelo de barrio que proponían ambos mundos no podía estar más distante pues pasamos desde una disposición de buena vecindad con pequeñas labores compartidas, en las periferias urbanas de Nueva Inglaterra, hasta los vastos equipamientos colectivos de las Colonias de Frankfurt (figura 12), aunque conocemos otros modelos estadounidenses más proclives a la socialización de determinadas tareas ${ }^{29}$. Catharine Beecher describe que "una pequeña iglesia, una escuela, y una vivienda familiar confortable pueden reunirse en un solo edificio, y por una suma de dinero muy moderada"30. En su Manual, hay una breve discusión del modelo de Barrio Cristiano, donde entre diez y doce familias pueden compartir lavandería y panadería.

La disolución de la ciudad central obligó a Ernst May, con su fuerte activismo social, a proporcionar todo tipo de servicios a las nuevas colonias, introduciendo la idea abstracta de barrio como un elemento independiente provisto de escuelas, piscinas, lavanderías, guarderías, restaurantes o centros comerciales. Asimismo, las viviendas mínimas requerían unos servicios centralizados que facilitasen las tareas domésticas. Por otro lado, ambos modelos inciden en su fuerte relación, por contigüidad física o dominio visual, con la naturaleza. La casa de Beecher contiene elementos adyacentes para mitigar los rigores del clima, como las piazzas y los conservatories. Sin embargo, para los alemanes, "luz, aire y sol" fue el lema del nuevo ideario constructor.

El aspecto que, sin lugar a dudas, es más evidente en esa transferencia de ideas a través del Atlántico en sentido Este es la racionalización de la vivienda, a través de la flexibilidad, la búsqueda de la dimensión mínima, el estudio de los procesos o la normalización de las técnicas constructivas (figura 13). En el caso americano, la combinación de salón y dormitorio, gracias al tabique móvil, otorga una gran flexibilidad a la casa. En Alemania, esto se lleva al extremo y su punto de partida es el diseño de cuartos-células. Su valor no se refiere a la medición de la superficie de la vivienda, sino al número de camas que contiene, en donde el lecho significa la unidad de medida para todas las necesidades (proporción del estar con el comedor, la cocina o el baño). Ya establecida esta ratio, se estudia una configuración distributiva que garantice los parámetros óptimos de luz solar, ventilación y aireación; resumiendo, un máximo de beneficio social con un mínimo de esfuerzo económico.

La cocina es un lugar clave, donde se reducen las dimensiones y, en cambio, se mejora su funcionamiento (figura 14). La cocina-máquina admite una disminución y compactación gradual, desde los $15 \mathrm{~m}^{2}$ de la propuesta por Beecher ${ }^{31}$, pasando por los 9,19 $\mathrm{m}^{2}$ de Frederick, para llegar a la pieza estandarizada de Frankfurt que en

29. Al respecto, véase HAYDEN, Dolores. The Grand Domestic Revolution: A History of Feminist Designs for American Homes, Neighborhoods, and Cities. Cambridge (Massachusetts): MIT Press, 1981.

30. Beecher y Beecher Stowe, op. cit., supra, nota 8, p. 455.

31. En su texto las Beecher hacen referencia a las cocinas compactas de las naves a vapor. Íbidem, p. 32. 
PROYECTO, PROGRESO, ARQUITECTURA

69
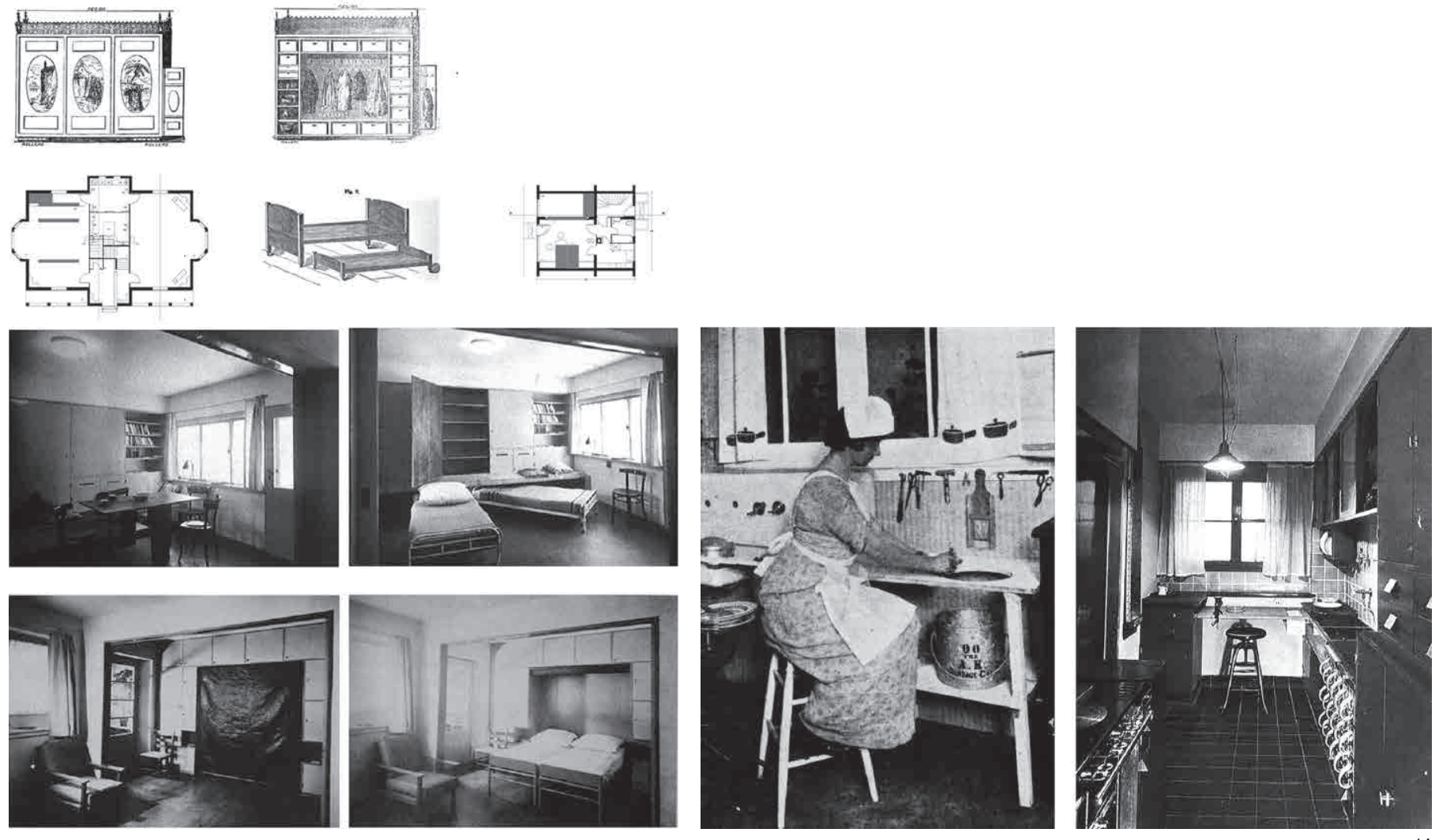

lograron el sueño americano con esta cocina de produc-

su primera fase alcanzó los 6,46 $\mathrm{m}^{2}$ y al final los 5,50 $\mathrm{m}^{2}$. Desarrollado en 1926 por Schütte-Lihotzky, el modelo Frankfurt sigue los principios de racionalización que aún perduran; con sus dimensiones de 1,90 x 3,40 metros y su disposición estándar, se reduce eficazmente el tiempo para elaborar una comida. Beecher ubica la cocina en el corazón de la casa y se propone como un equipo técnico que canaliza además el calor y la ventilación. Algo similar ocurre en Frankfurt, aunque ese espacio sea más eficaz, pues se transforma en un aparato, una instalación, una máquina para la vivienda mínima.

Para una mejor racionalización del proceso de trabajo, Frederick define los diagramas de ahorro en circulaciones (pasos) y de longitud de trayectoria. Propone nuclearizar la cocina, con zonas diferenciadas para cada proceso, con su respectiva mesa, estantería, almacenaje y utensilios específicos. Sin embargo, en Frankfurt, May y Schütte-Lihotzky consideraron necesario incluir todo el mobiliario de la cocina durante la construcción de la vivienda, para poder ser financiado y repercutir ese coste en el alquiler. En cierto sentido, los alemanes ción en serie.

La normalización del mobiliario se restringe, en el caso estadounidense, a manuales técnicos y de utilización estándar, del tipo Hoosier cabinet, que destaca por ser compacto, modular y de múltiples accesorios, o al armario para lavar y planchar incluso con su manual de instrucciones precisas para quitar manchas. Para May, la vivienda, como artículo de masas, sólo podía edificarse con criterio y rigor económico si estaba tipificada, si sus elementos se normalizaban y si el proceso constructivo se mecanizaba. El concepto básico de racionalización de la vivienda implicaba homologar desde picaportes, puertas o ventanas hasta techos y cubiertas, aunque también incluía el mobiliario combinable. Estas normas fueron formalizadas en el Registro de Frankfurt y debían ser aceptadas por todos los contratistas.

Por último, el equipo de Frankfurt comprende que la divulgación acometida por las norteamericanas mediante exposiciones, conferencias, libros, revistas y cintas de cine había sido especialmente eficaz a la hora 
15. Izquierda: Fotograma de una de las películas originales de Frank y Lillian Gilbreth, donde se aplica el concepto de Time-Motion Studies, 1913-17. Derecha: Imagen del film dirigido por Paul Wolff: Neues Bauen in Frankfurt am Main, 1928.
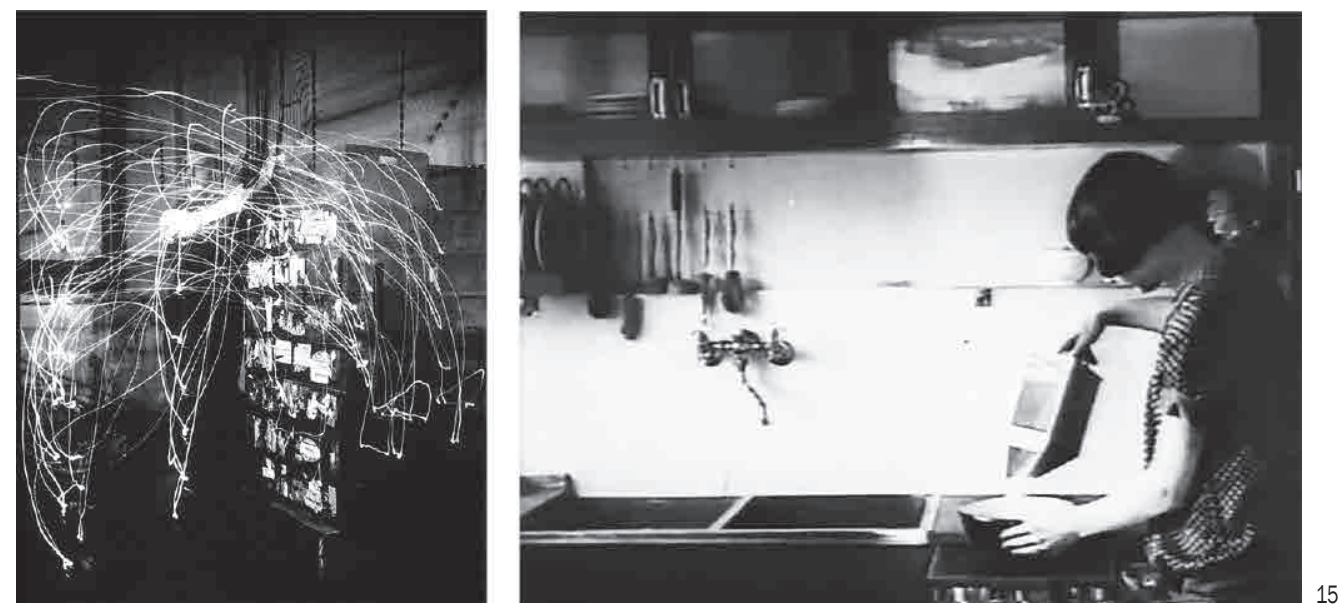

de concienciar al ama de casa. En otoño de 1926, May fundó la revista mensual El nuevo Frankfurt ${ }^{32}$, donde documentaba la actividad edificatoria y así lograba un foro internacional de debate sobre la nueva construcción y el arte de vanguardia. Schütte-Lihotzky escribió un sinfín de artículos centrados en sus temas de investigación: la cocina de Frankfurt, cocinas de colegios o para la enseñanza, y viviendas para la mujer trabajadora. En ellos argumentaba las mejoras de la racionalización ${ }^{33}$ sobre la economía doméstica, como obtener más tiempo para el ocio, la cultura o la educación de los hijos. También explicaba que la producción en serie de las viviendas reducía significativamente los alquileres y que la vivienda mínima exigía unos servicios centralizados que facilitasen las tareas domésticas, tales como lavanderías automatizadas con equipamiento moderno y jardines de infancia. Su propósito último era hacer comprender a las mujeres que debían reivindicar mejores viviendas ya que ellas eran las primeras afectadas y para ello debían superar las convenciones en la organización del hogar.

En cuanto a exposiciones y foros de debate, además de Frederick con sus conferencias por los circuitos europeos, Lillian Gilbreth participa profusamente en Congresos en Europa durante los primeros años veinte, en donde aporta la mayor parte de la nueva terminología sobre la eficiencia en la gestión. Después, en 1931, Lillian exhibe la "cocina funcional" junto a la "cocina de Frankfurt" de Schütte-Lihotzky, en la Exposición de Construcción de Berlín. Previamente, en marzo de 1926, esta última había retransmitido por radio una célebre conferencia ${ }^{34}$, en la que disertó sobre sus ideas respecto al ahorro de trabajo en la organización de la casa (figura 15).

A lo largo del documental promocional Neues Bauen in Frankfurt am Main ${ }^{35}$, se observa el fenómeno de la construcción de vivienda en la ciudad. En la sección

32. Ernst May: DAS NEUE FRANKFURT: Fünf Jahre Wohnungsbau in Frankfurt am Main. Frankfurt: Henrich Editionen, 2011. Facsímil de los números de la revista internacional Das Neue Frankfurt.

33. KAES, Anton; JAY, Martin and DIMENDBERG, Edward. The Weimar Republic Sourcebook: "Grete Lihotzky. Rationalization in the Household", University of California Press, Berkeley, 1994, pp. 462-465.

34. El título de la conferencia retransmitida fue “Arbeitssparende Haushaltsführung”. NOEVER, Peter (ed.). op. cit., supra, nota 8, p. 75.

35. Dirigida por Paul Wolff en 1928, la película está dividida en cuatro secciones: La vivienda mínima; La cocina Frankfurt; Una nueva forma de construir; y La vivienda prefabricada. 
dedicada a la cocina, se equiparan un ejemplo convencional de la época y la proyectada por Lihotzky. Los títulos y las secuencias del film enfatizan lo poco práctico de la vieja cocina y las ventajas del impecable nuevo diseño. Hacia el final, mediante un diagrama de movimiento similar al ideado por Frederick, se demuestra su eficacia. Por su parte, las películas de los Gilbreth serían utilizadas como método científico de análisis y registro visual, insistiendo en las áreas de posible mejora. Al mismo tiempo, se proponían como medio para capacitar a los operarios en la correcta ejecución de una tarea. De igual manera, las cintas alemanas se enfocaban hacia una forma didáctica de enseñar a las mujeres a habitar sus nuevos hogares, aunque también contenían un alto nivel de propaganda para difundir el óptimo resultado de Frankfurt am Main.

\section{PERMANENCIAS. SINGLADURA DE LO DOMÉSTICO.}

Desde posiciones muy diversas, la intelectualidad expuesta en "El hogar de la mujer americana", el pragmatismo de "La nueva organización doméstica" y el cientifismo de "La psicología de la gestión", todos esos manuales tipificaron, hicieron eficiente y optimizaron, respectivamente, la concepción proyectual y productiva de la vivienda. Esto implicaba racionalizar, modernizar y mecanizar su contenido con el fin de mejorar el trabajo y la vida en su interior. En los compendios, no se cuestionaba si el lugar apropiado para la mujer era el hogar, sino que sólo se afirmaba que no estaba bien ideado. Estos manuales fueron el lugar inicial donde aparece la casa desde dentro, desde el bienestar, el placer de habitar, lo que ha dejado rastro hasta nuestros días, pues, por primera vez, la casa es pensada desde un nuevo punto de vista: el del usuario.

Como queda patente, la influencia de las ingenieras americanas es capital para entender los avances en la vivienda social moderna europea que May o SchütteLihotzky desarrollaron. El eco de las ideas de aquellas pioneras todavía reverbera tenaz en múltiples detalles, desde el tipo de ciudad pretendida, hasta una definición normalizada del mínimo elemento. Lo novedoso ha sido descubrir la singular estela de sus trabajos y, lo principal, el modo en que esos avances entraron en el grupo de May, por medio de las asociaciones femeninas. Estas mostraron las distintas corrientes estadounidenses a Lihotzky e inocularon la idea de que las condiciones domésticas debían estar sujetas al mismo tipo de escrutinio racional, generalmente reservado para la optimización de la productividad, por lo que racionalización y liberación se equipararon en la mente de Grete.

Durante la entreguerra, lo cotidiano, lo ordinary de los manuales femeninos, se asumió con normalidad al proyectar viviendas colectivas. Bien podría ser la mujer la causante de la cultura de masas, del acervo popu$\operatorname{lar}^{36}$. Incluso se insinúa metafóricamente que la cocina es el enclave de la producción de la cultura de masas, el epicentro de la banalidad popular en la emergente cultura de consumo y que tiene su apogeo en los años cincuenta. Quizás en las Bellas Artes se haya visto con más frecuencia, pero en la arquitectura puede que sea la primera vez que un arte inferior, el low brow inglés, haya interferido en la alta cultura, en el high brow. Durante los dos últimos siglos, y quizá menos en el actual, la mujer ha ocupado un lugar como fabricante y receptora de artes menores, ligadas a la emoción subjetiva, a la pasividad romántica. Cierta modernidad, en algún sentido, repudió la cultura de masas, distanciándose de todo aquello trivial o banal de la vida diaria, al refugiarse en un idealismo aristocrático, alejado del pulso cotidiano. La adscripción universal de la feminidad a la cultura de masas siempre ha dependido de su exclusión real de la alta cultura y sus estamentos.

De la mano de estas investigadoras es posible descubrir una nueva mirada, íntegra pero irreverente, libre de prejuicios, que prima el valor de lo próximo, lo inmediato, el instante perdurable. La importancia de lo doméstico, lo cotidiano, hace que podamos especular más sobre el interior vital que sobre la apariencia periférica. Además, por tratarse de una aspiración íntima del ser humano, la casa se adscribe a la cultura de la publicidad y del consumo, para situarse equidistante entre el progreso técnico y los cambios de modo de 
vida $^{37}$. El rol contemporáneo de la mujer, su relevancia global, su expresión femenina, asociados inevitablemente con la domesticidad, desplazan la atención proyectiva desde el exterior del edificio hacia el interior. Vemos claro que la vigencia de lo doméstico a través de los procesos de uso $^{38}$ y los objetos de la domesticidad ${ }^{39}$ de aquellas "ladys" persiste en nuestro modo de entender el habitar, ya que existió la voluntad de encontrar la felicidad a través de una cierta idea de lo doméstico que trata de reconciliar un modelo tradicional con los avances, ventajas y comodidades modernos.

\section{Bibliografía citada:}

BAUDRILLARD, Jean. Le système des objets. Paris: Éditions Gallimard, 1968.

BEECHER, Catharine. A Treatise on Domestic Economy, for the Use of Young Ladies at Home and at the School. Boston: Marsh, Capen, Lyon y Webb, 1841.

BEECHER, Catharine, BEECHER STOWE Harriet. The American Woman's Home or Principles of Domestic Science Being a Guide to the Formation and Maintenance of Economical Healthful Beautiful and Christian Homes. New York: J. B. Ford and Co., y Boston: H. A. Brown \& Co., 1869.

BEHNE, Adolf. Der moderne Zweckbau. Vienna/Berlin: Drei Masken Verlag, 1923. Traducido al español 1923. La construcción funcional moderna. Barcelona: Ed. del Serbal, 1994.

BERNĖGE, Paulette. Vers une cuisine automatique. En: Mon Chez Moi. Fascículo IV, 1 de Octubre 1923 - 1 de Noviembre 1923, pp. 25-29.

BOOT, Marjan y CASCIATO, Maristella (ed.). La casalinga riflessiva. La cucina razionale come mito domestico negli anni '20 e '30. Catálogo de Exposición, Octubre-Noviembre 1983, Palazzo delle Esposizioni. Roma: Multigrafica Editrice, 1983.

ECO, Umberto. El vértigo de las listas. Barcelona: Ed. Lumen, 2009.

ECO, Umberto. Apocalípticos e Integrados. Barcelona: Ed. Lumen, 1965.

ELSAESSER, Thomas. The Camera in Kitchen. Grete Schütte-Lihotzky and Domestic Modernity. En Christiane SCHÖNFELD, ed. en colaboración con Carmel FINNAN, Practicing Modernity: Female Creativity in the Weimar Republic. Würzburg: Königshausen \& Neumann, 2006, pp. 27-49.

Ernst May und das neue Frankfurt 1925-1930, Catálogo de la Exposición en el Deutschen Architekturmuseum, Frankfurt am Main. Berlin: Wilhelm, Ernst \& Sohn, 1986.

Ernst May: DAS NEUE FRANKFURT: Fünf Jahre Wohnungsbau in Frankfurt am Main. Frankfurt: Henrich Editionen, 2011.

FREDERICK, Christine. The New Housekeeping: Efficiency Studies in Home Management. Garden City (New York): Doubleday, Page \& Company, 1913. FREDERICK, Christine. Household Engineering: Scientific Management in the Home. Chicago: American School of Home Economics, 1920.

FREDERICK, Christine. Le Taylorisme chez soi. Pratique de la direction de la maison. Paris: Dunod Editeur, 1920.

37. ELSAESSER, Thomas. The Camera in Kitchen. Grete Schütte-Lihotzky and Domestic Modernity. En: Christiane SCHÖNFELD, ed. y Carmel FINNAN, Practicing Modernity: Female Creativity in the Weimar Republic. Würzburg: Königshausen \& Neumann, 2006, pp. 27-49.

38. ECO, Umberto. El vértigo de las listas. Barcelona: Ed. Lumen, 2009.

39. BAUDRILLARD, Jean. Le système des objets. Paris: Éditions Gallimard, 1969. 
FREDERICK, Christine. Die rationelle Haushaltführung. Betriebswissenschaftliche Studien. Berlin: Julius Springer Verlag, 1921. FREDERICK, Christine. Naukowa organizacja w gospodarstwie domowen. Warsaw: Nakladem Instytuta Naukowej Organizacji, 1926. FREDERICK, Christine. De Denkende Huisvrouw. Haarlem: Tjeenk Willink, 1928. FREDERICK, Christine y TEALDY, Lorenzo. La donna e la casa. II taylorismo della vita domestica. Torino: C. Accame, 1928. FREDERICK, Christine. Selling Mrs. Consumer. New York: The Business Bourse, 1929.

GIEDION, Sigfried. Mechanization Takes Command: A contribution to Anonymous History. New York: Oxford University Press, 1948.

GILBRETH, Lillian. The Psychology of Management: The Function of the Mind in Determining, Teaching and Installing Methods of Least Waste. New York: Sturgis \& Walton Company, 1914.

GILBRETH, Frank B. y GILBRETH, Lillian. Fatigue Study: The Elimination of Humanity's Greatest Unnecessary Waste. A First Step in Motion Study. New York: Sturgis \& Walton Company, 1916.

GILBRETH, Frank B. y GILBRETH, Lillian. Ermüdungsstudium (Fatigue Study). Eine Einführung in das Gebiet des Bewegungsstudiums. Berlín: Verlag des Vereines Deutscher Ingenieure, 1921.

GILBRETH, Lillian. Verwaltungspsychologie. Berlin: Verlag des Vereins dt. Ingenieure, 1922.

GINZBURG, Moisei. Stil'i epokha, Moskva: Gosizdat, 1924. Traducido al inglés por Anatole Senkevitch, Jr. Style and Epoch. Cambridge (Massachusetts): MIT Press, 1982.

GROPIUS, Walter. Die Kunst In Industrie Und Handel. Jena: Verlag Eugen Diederichs, 1913.

HAYDEN, Dolores. The Grand Domestic Revolution: A History of Feminist Designs for American Homes, Neighborhoods, and Cities. Cambridge (Massachusetts): MIT Press, 1981.

LE CORBUSIER-SAUGNIER. Vers une architecture. Paris: Les Editions G. Cres et Cie., 1923. Traducido al español Hacia una arquitectura. Barcelona: Ediciones Apóstrofe, 1977.

MCLEOD, Mary. "Architecture or Revolution: Taylorism, Technocracy, and Social Change”. Art Journal, vol. 43, n. ${ }^{2}$ 2, pp.132-147.

MENDELSOHN, Erich. Amerika: Bilderbuch eines Architekten, Berlin: Rudolf Mosse Buchverlag, 1926. Versión inglesa: Erich Mendelsohn's "Amerika”: 82 Photographs, New York: Dover Publications Inc., 1993.

MEYER, Erna. Der neue Haushalt ein Wegweiser zu wirtschaftlicher Haushaltsführung. Stuttgart: Franckh'sche Verlagshandlung, 1926.

NOEVER, Peter (ed.). Margarete Schütte-Lihotzky. Soziale Architektur Zeitzeugin eines Jahrhunderts, Wien: Bóhlau, 1993.

SCHÜTTE-LIHOTZKY, Margarete. Rationalisierung im Haushalt. En: Das Neue Frankfurt, n.5, 1926-1927, pp.120-130. Traducido al inglés en KAES, Anton; JAY, Martin and DIMENDBERG, Edward. The Weimar Republic Sourcebook. Grete Lihotzky. Rationalization in the Household. Berkeley: University of California Press, 1994.

TAUT, Bruno. Die Neue Wohnung. Die Frau als Schöpferin. Leipzig: Klinkhardt \& Biermann, 1924.

Carmen Espegel Alonso (Palencia, 1960). Doctora Arquitecta por la Escuela Técnica Superior de Arquitectura de la Universidad Politécnica de Madrid, donde ejerce como Profesora Titular del Departamento de Proyectos Arquitectónicos. A nivel profesional, en 2002 crea junto a Concha Fisac la firma espegel-fisac architectos. Su orientación en la investigación se centra principalmente en el campo de la vivienda y de la mujer en la arquitectura. Dirige el Grupo de Investigación en Vivienda Colectiva GIVCO UPM-ETSAM. Entre sus publicaciones se encuentran Women Architects in the Modern Movement (2018), Collective Housing 1992-2015 Vol.II (2016) y 1929-1992 Vol.I (2013), Eileen Gray: Objects and Furniture Design (2013), Aires Modernos, E.1027: Maison en bord de mer de Eileen Gray y Jean Badovici (2010) y Heroínas del espacio (2008).

Gustavo Rojas Pérez (Granada, 1980). Arquitecto por la Universidad de Granada. Cofundador y socio de Architectural Matter. Master in Collective Housing por la Universidad Politécnica de Madrid. Profesor contratado (L.D. Ayudante 2010-2015) en el Departamento de Proyectos Arquitectónicos de la UPM-ETSAM. Miembro del Grupo de Investigación en Vivienda Colectiva GIVCO UPM-ETSAM. Participación en dos proyectos competitivos de Plan Nacional de I+D+i (Atlas de Vivienda Colectiva Española S.XX y Atlas de Vivienda Colectiva Contemporánea Europea) y en otro fruto de un convenio de investigación con el Área de Urbanismo y Vivienda del Ayuntamiento de Madrid (Tipos existentes en el área central de Madrid M-30 y su potencial de adaptación en vivienda contemporánea). 
LA ESTELA DE LAS INGENIERAS DOMÉSTICAS AMERICANAS EN LA VIVIENDA SOCIAL EUROPEA THE TRAIL OF AMERICAN DOMESTIC ENGINEERS IN EUROPEAN SOCIAL HOUSING

Carmen Espegel Alonso, Gustavo Rojas Pérez

p.59 With the technological advances of the beginning of the twentieth century, Europe's most relevant architects, Gropius $^{1}$, Behne $^{2}$, Le Corbusier ${ }^{3}$, Ginzburg ${ }^{4}$ and even Mendelsohn ${ }^{5}$, turned to with admiration the United States regards to its industrial mass production and work efficiency methods. They all saw their theoretical concerns reflected in Taylor's and Ford's proposals. Therefore, they tried to transfer these successes to modern architecture, since, in a way, they were basic components of social renovation ${ }^{6}$.

At the same time, the elite, left-leaning, European intelligentsia-Bruno Taut or Ernst May in his conception of the house for the working class - also turned their eyes to America, but in their case focusing on research carried out by non-professionals. Thanks to the work of Margarete Schütte-Lihotzky ${ }^{7}$, the Manuals written by Catharine Beecher, Christine Frederick and Lillian Gilbreth (figure 1) were hugely influential in the idea of domesticity applied to the residential programs carried out by the New Frankfurt initiative led by Ernst May ${ }^{8}$ between 1925 and 1930.

In the following pages we will discover the extraordinary paths by which these American explorations reached the European context, outside the usual circles of architectural theory. German professionals, from the stance of the social responsibility that permeated their work and with an open mind towards public participation, established a conversation with women's associations that, eventually, introduced American progress into the modernization of

p.61 interwar housing. As we shall see, what started off as compendia aimed at the world of women ended up becoming the leitmotiv of social housing pioneers.

\section{PROFILES. AN INTELLECTUAL, PRAGMATIC AND SCIENTIFIC APPROACH}

Catherine Beecher (1800-1878) founded the Hartford Female Seminary in 1823, a school for young ladies that went beyond traditional education by establishing a course plan that even included science theory and physical activities. Her rationalist spirit, along with her sensitivity when it came to observe reality, led her to write several books. Among them A Treatise on Domestic Economy, for the Use of Young Ladies at Home and at the School ${ }^{9}$ stands out, considered the first ever published complete guide to home maintenance in the United States.

Her following book. The American Woman's Home or Principles of Domestic Science Being a Guide to the Formation and Maintenance of Economical Healthful Beautiful and Christian Homes ${ }^{10}$ was coauthored with her sister Harriet Beecher Stowe ${ }^{11}$ (figure 2). This document provides a rigorous, step by step, description of all house chores, explaining them in full detail; the term mechanization became the key term for the "professional" housewife. The cover of the book portrays her ideal world: home, family and church. But this is an enlightened home, where all of its members read under an efficiently designed lamp that glows softly towards the ceiling but casts an intense light onto the reading surface. This is a book to tackle life and its organization, with the family at its core.

The authors' efficient transformation of the house revolves around a central spine (figure 3), a technical feature that accommodates not only staircases, closets, the toilet, kitchen and stove but also the heating ducts that distribute hot air from a boiler in the basement to all quarters as well as a ventilation shaft with entry and exit grilles for air

p.62 renovation ${ }^{12}$. Spatial improvements are also included, with rooms that can be transformed by means of movable closets and sliding panels aimed at reducing the size of the house not only for economic reasons, but to make its use, care and maintenance more efficient.

However, there is a major difference between these proposals and Beecher's prior designs: the intentional "undefined" use of the rooms of the house. Their furniture — the piano, the bed-qualify the exact use of each quarter. Flexibility is maximized with decorative furniture-screens that hide the dressing room or extra beds. Besides this, the kitchen, with a worktop at a continuous level, becomes a space for rational processes where two areas are made distinct: one for cooking at the stove and another for food preparation.

The truth is that Beecher did not write simple manuals or kitchen anthologies, but thoroughly thought out treatises, comprehensive text books that recalled the spirit of the eighteenth-century enlightenment ${ }^{13}$ showing reasoned knowledge and a first systematic approach to home economics, all for the sake of promoting a healthy family environment.

In order to get to know our next personality, Christine Frederick (1883-1970), we may as well use the same words she applied in Selling Mrs. Consumer ${ }^{14}$ to describe her broad professional profile: counsellor to manufacturers on marketing home goods; founder of first advertising women's organization; first to apply the principles of scientific management to home. She graduated from Northwestern University and dedicated her life to teaching. Later on and thanks to her husband's business partners she attained firsthand knowledge of the scientific organization of work. With their help, she tried to apply these principles to the home, with all of the shortcomings this involved, since in factories there are just a few types of processes and they are endlessly repeated while in the home there is an infinite amount of chores to carry out and they overlap. That is, there was a problem of scale. In 1912 she began to explain all of this to middle class housewives in the pages of the Ladies' Home Journal. 
Her texts advocate for more efficiency in the kitchen, contending that housewives had the right to enjoy the proper tools and to experiment with up-top-date technologies, just like businessmen and farmers did. To defend her theories, Frederick set up a model kitchen, the Applecroft Home Experiment Station, in her own home in Long Island (figure 4). In this way, her house became a laboratory in which she was both the researcher and the object of research. This experiment led to a lengthy practical investigation that lasted from 1910 to 1930.

The book The New Housekeeping: Efficiency Studies in Home Management ${ }^{15}$ is a compilation of the texts she published in installments in The Ladies Home Journal. Scientific management meant efficiency and this was achieved by introducing new household appliances, which by then were more affordable. This manual includes her well-known diagrams showing the incorrect and correct use of the kitchen, the difference between which lies in the position of the furniture and the appliances. She differentiates between two circuits: a clean one that includes the exit of prepared food towards the dining room and a dirty one with the dishes that need to be washed (figure 5).

Her following compendium Household Engineering: Scientific Management in the Home ${ }^{16}$ includes a detailed description of chores, which she enumerates, analyzes, reflects upon and programs to, in the end, redefine in more efficient terms (figure 6). Her argument in favor of domestic utensils or mechanical equipment has to be understood from the point of view of a house without household help. She dignified housework by simply choosing the right utensil for each task since, having previously defined cleaning methods, she understood that if the correct tool was used, work could be made easier and less tiresome for the housewife.

The pragmatism that impregnated all of her analyses stands out. They led to the introduction of standard heights for counters and encouraged improvements in kitchen design in order to avoid unnecessary to-and-fros. Her texts embraced publicity as a means of informing women of the benefits of this new era. By taking scientific principles into the home her work also incorporated the domestic realm into an industrial system that was reshaping both business and politics. Her major contribution was the change of perception that led to the integration of the lives of homemakers in the new economic and social order.

The third protagonists of this story are the Gilbreths, Lillian (1878-1972) and Frank (1868-1924). They are known for pioneering scientific management with what they called Time-Motion Studies, where they used cinematic recordings to study processes and production speeds. In 1914, Lillian published The Psychology of Management: The Function of the Mind in Determining, Teaching and Installing Methods of Least Waste ${ }^{17}$, in which she discusses the well-being of the work environment, labor incentives and accident prevention. The gradual suppression of unnecessary fatigue, be it physical or psychological, led her to write, Fatigue Study: The Elimination of Humanity's Greatest Unnecessary Waste; A First Step to Motion Study ${ }^{18}$, in 1916.

The key to their method consisted in marking out the space, measuring time and filming work processes to later analyze and optimize these parameters (figure 7). Inspired by Taylor's Time Study Work technique and its implementation of standard times, the Gilbreths, in their Motion Study Work, proposed new technological terms, which made it easier to carry out an analysis of operative processes with a scientific perspective. The couple used their technical knowledge to develop a method based on the thorough analysis of movements, which in part consisted of the detailed filming of the actions of workers and their body posture. Their films ${ }^{19}$ show various industrial processes on the basis of which the study of movement technique was developed.

Their interest in a rationalized analysis of time involved being able to visualize it adequately with a series of dynamic diagrams. In this sense, in the "Motion Efficiency Study" (figure 8) the Gilbreths held a light bulb to the back of a hand of an female worker and they used slow exposure times to photograph the path of movement as if it were a line of continuous light in space.

After Frank's death in 1924, Lillian continued with their work, but she focused on her area of expertise, the psychology of management ${ }^{20}$. She highlighted the importance of the "human factor" - the precursor of ergonomicsin the application of scientific techniques and she proved to managers that production rose with better lighting and breaks throughout the workday. Besides this, she took these observations to the realm of the home, proposing improvements in appliances, the design of the "efficient kitchen" and, later on, the first kitchen adapted to the needs of the handicapped and people with heart disease.

\section{REFERENCES. CHRONICLE OF A JOURNEY OF IDEAS}

As mentioned, the most important European architects of the time admired the United States for its industrial production and manufacturing efficiency methods. Taylor, with his time study, and Ford and his assembly line, recalled concepts such as efficiency, perfection, economy, productivity and precision. These ideas inspired European professionals who strove to take these industrial achievements to the realm of modern architecture. Factories, silos or assembly lines were concrete images of a market system, American capitalism, that prioritized mass production. 
However, Europe assimilated the work of these female pioneers as references in the field of interwar inhabitation. In 1924, Bruno Taut published Die Neue Wohnung: Die Frau als Schöpferin ${ }^{21}$ in Berlin. It can be translated as The New Dwelling, but its subtitle mentions the woman as the designer, or more literally, as the "creator". At the beginning of the book he mentions Frederick and her manual The new housekeeping, including the circulation diagrams that improved the organization of living space.

Given their close friendship, it is reasonable to believe that it was Bruno Taut who introduced Christine Frederick's p.66 publications to Margarete Schütte-Lihotzky. But we think that there is a more plausible connection involving Erna Meyer $^{22}$, who advised J.J.P. Oud in the kitchen design for the Weissenhof (1927) ${ }^{23}$ housing project, and Irene Witte, who faithfully translated Frederick's and the Gilbreth's studies into German. These two women, with their ties to the network of women's associations and their knowledge of the advanced ideas put forward by the three American researchers, may well have shown Lihotzky the trends regarding efficiency in the home that came from the other side of the Atlantic. The spreading of these transfers from the United States and Europe took place in several formats: the translation of the manuals, articles in women's magazines, conferences throughout the Old Continent and the dissemination of their work through women's associations, which were on the rise during those years. (figure 9)

Pioneering this movement, as well as the fight against slavery, Harriet Beecher Stowe travelled to England in 1853, and later toured Paris and Rome. During those trips, she presented not only her own work, but also her sister's Treatise on Domestic Economy. However, Christine Frederick played a more important role in the spreading of these ideas given the publication of her works in the main European capitals ${ }^{24}$. Besides this, during the 1920 s, she gave many conferences ${ }^{25}$ about scientific progress in the United States mainly to European women's associations, with whom she had close ties. On her part, Lillian Gilbreth participated in multiple gathering ${ }^{26}$ presenting her studies about movement. Her friend, Irene p.67 Witte, who studied Taylor and work efficiency methods, translated her articles and books into German ${ }^{27}$.

Among the magazines of the time, the most relevant was Mon chez moi. La revue d'organisation ménagère, published by Paulette Bernège between 1923 and 1930. As its editor, she wrote the article "Vers une cuisine automatique" 28 , in clear reference to Le Corbusier's famous book. In it, she addressed the appliances used in American kitchens, using diagrams that were similar to Frederick's, tracing steps and distances, and even including some of the original plans and diagrams belonging to the American researcher.

After the end of the Great War, and thanks to the establishment of universal suffrage by the Weimar Republic, there was an exponential increase in the number of women's associations (figure 10): their newfound right to vote made them visible and decisive. This was also the case in other parts of Europe-Poland, Austria, the Netherlands and Belgium - where these federations proliferated, regardless of their religious, conservative, feminist, or political inclinations, and of their makeup, students, workers or housewives. These groups promoted the social influence as well as the education of women and defended their rights. Thanks to these networks, knowledge of these nascent American ideas was channeled and put into practice in the home.

\section{TRANSFERS. CIVIC AND CULTURAL REPERCUSSIONS}

Let us now analyze some of the aspects of the American model and how they were applied in the German context. In the first place, there was the way the city was conceived in the United States with its strong ties between housing and nature, in contrast with the Siedlungen, which were closer to the English model of the garden city (figure 11). Towards the end of the 18th century, Thomas Jefferson strived to create a diagram of the ideal democratic space, with the aim of promoting family farm units, leading to sprawl instead of the more compact model of New England cities. At the time, the debate revolved around the ideal urban model for the entire nation. In this context, Jefferson's agrarian utopia was not impartial, since it favored the dominance of a certain social class represented by the more traditional South. In general, bourgeois families abandoned the center of cities and established themselves in suburban homes. The first suburban prototypes designed by Catharine Beecher were conceived as spaces for female domestic work at the service of the family.

In contrast, for Frankfurt, Ernst May proposed growth around multiple centers, creating several satellite developments that functioned as self-sufficient, practically autarchic colonies. The idea was that these settlements p.68 should be established in the vicinity of nature, separated from the traditional city center by a green ring. Their general layout was based on the repetition of rows of houses, strictly along an East-West orientation, although there were variations depending on the topography and the road network. The 15,000 housing units built under May's guidelines represented more than $90 \%$ of the homes built and finished during that time. That impressive figure could not have been reached without the efforts put into economic efficiency from the design to the building phases.

The neighborhood model proposed on each continent could not have been more different: on the one hand, in the urban peripheries of New England, neighborly well being was promoted by sharing small chores, and on the other, in the Frankfurt Colonies, vast collective facilities were established (figure 12). However, other American models existed that were more open to the socialization of certain tasks ${ }^{29}$. Catharine Beecher states that "a small church, a school-house, and a comfortable family dwelling may all be united in one building, and for a very moderate sum" 30 . In her manual there is a brief description of a model Christian Neighborhood, where some ten to twelve families could share a laundry and a bakery.

The dissolving of the central city led Ernst May and his forceful social activism to make all sorts of services available in the colonies, introducing the abstract notion of neighborhood as an independent entity with schools, 
swimming pools, laundries, kindergartens, restaurants and shopping centers. Likewise, the minimum dwellings he advocated required a series of centralized services to facilitate domestic chores. Besides this, both the American and German models underlined the need for a strong relationship with nature due to its physical proximity and visual dominance. Beecher's house included adjacent features that mitigated the rigors of climate, such as piazzas and conservatories. However, for the Germans, "light, air and sun" was the motto of their innovative building principles.

The aspect that, without a doubt, is most evident in this transfer of ideas eastwards across the Atlantic is the rationalization of the dwelling — through flexibility — the search for minimum sizes, the study of processes and the standardization of construction techniques (figure 13). In the American case, the combination of the living room and bedroom thanks to movable partitions made the housing unit very flexible. In Germany, this was taken to extremes with the design of room-cells as a starting point. The value of a house did not reside in how much surface area it had, but in how many beds it could accommodate, where the dimension of the cot was the measuring unit for the rest of needs (the proportion of the living area with respect to the dining room, kitchen and bathroom). Having established this ratio, a layout was studied that guaranteed optimal parameters as far as natural light, ventilation and airing were concerned. All in all, a maximum social benefit at a minimum economic cost.

The kitchen is a key player in all of this; its size is reduced but, in exchange, its functionality is improved (figure 14). The kitchen-machine allows for the reduction in its dimensions and its gradual compactness, ranging from Beecher's $15 \mathrm{~m}^{2}$ proposal $^{31}$ to Frederick's 9,19 $\mathrm{m}^{2}$, to become the standardized 6,46 $\mathrm{m}^{2}$ Frankfurt unit in the first phase of the design, which ended up going down to 5,50 $\mathrm{m}^{2}$. Developed in 1926 by Schütte-Lihotzky, the Frankfurt model follows standardized principles that are still applied today; with its 1,90 by 3,40 meters and its standard layout, the time needed to make a meal is efficiently reduced. Beecher places the kitchen at the heart of the house and it is understood as a technical feature that also channels heating and ventilation. Something along similar lines occurs in Frankfurt, however, this space becomes even more efficient, as it is turned into an appliance, an installation, a machine for the minimum dwelling.

In order to improve the streamlining of the work process, Frederick defines diagrams that save on circulation (steps) and reduce the distance of trajectories. Her proposal is to create nucleuses in the kitchen, with differentiated areas for each process, with their own specific table, shelf, storage units and utensils. However, in Frankfurt, May and Schütte-Lihotzky considered the incorporation of all kitchen furniture a necessity in the construction of the housing unit, in order to finance it and include its costs in the rent. In a sense, the Germans made the American dream come true with this mass produced kitchen.

The normalization of furniture was limited, in the American case, to technical manuals and standard uses, like the Hoosier cabinet, which stood out for being compact, modular and for containing several accessories, or the washing and ironing closet that included specific stain removing instructions. For May, the dwelling, as an article for the masses, could only be built following strict economic criteria if it was typified, if its elements were normalized and its construction process was mechanized. The basic concept behind the rationalization of the dwelling involved homologating everything ranging from handles, doors and windows to ceilings and roofs, even though furniture combinations were also made available. These standards were included in the Frankfurter Register and had to be accepted by all contractors.

Lastly, in order to raise awareness of these issues among housewives, the Frankfurt team understood how efficient the dissemination of the ideas of the Americans had been, with exhibitions, conferences, books, magazines and movies. In the fall of 1926, May established the monthly publication The New Frankfurt ${ }^{32}$, which documented all of the ongoing building activities and therefore created an international debate forum regarding new construction techniques and avant-garde art. Schütte-Lihotzky wrote many articles focusing on her research themes: the Frankfurt kitchen, kitchens for schools and for teaching and housing for the working woman. In these writings ${ }^{33}$ she extolled the improvements that domestic economy rationalization provided, such as more time for leisure, culture or the education of children. She also explained how the mass production of houses significantly reduced rent costs and that minimum dwelling required centralized services, which made domestic chores much easier, such as automated laundromats with modern appliances and kindergartens. Her ultimate goal was to make women understand that they had to demand better homes since they were the most affected by the design of these spaces, and in order to do so they had to overcome conventionalities regarding the organization of the house.

As for exhibitions and debate forums, besides Frederick with her conferences in European circuits, Lillian Gilbreth participated profusely in congresses throughout the continent during the first part of the 1920s, bringing with her most of the new terminology used in efficient management. Later on, in 1931, Lillian showed the "functional kitchen" along with Schütte-Lihotzky's "Frankfurt kitchen" at the Berlin Building Exhibition. Before that, in March 1926, the German designer had aired a well known conference on the radio ${ }^{34}$, in which she discussed her ideas about saving work time and effort in the organization of the home (figure 15).

The building effort that took place in the city of Frankfurt can be appreciated throughout the promotional documentary Neues Bauen in Frankfurt am Main ${ }^{35}$. In the part dedicated to the kitchen, a conventional space is compared to the one Lihotzky designed. The titles and film sequences highlight just how unpractical the traditional kitchen was and the advantages of the impeccable new design. Towards the end of the piece, with the use of a circulation diagram similar to the one created by Frederick, its efficiency is proven. On their part, the Gilbreth's movies were used as a scientific analytical method and as a visual register pinpointing areas were improvements could still be 
made. They were also used to train workers in the correct execution of tasks. Likewise, the German films focused on didactic ways of teaching women how to inhabit their new homes, even though they also were highly propagandistic in their spread of the successful results of Frankfurt am Main development.

\section{PERMANENCES. THE COURSE OF DOMESTICITY}

From their diverse stand points, the intellectuality of The American Woman's Home, the pragmatism of The New Housekeeping and the scientism of The Psychology of management, all of these manuals typified, made more efficient and optimized, respectively, the design and productive approach to the house. This meant rationalizing, modernizing and mechanizing its content with the goal of improving work and life within it. In these compendia, whether the adequate place for the woman was the house or not was not put into question, they only stated that its spaces were not well thought out. These manuals were the first to show the house form the inside, through the lens of well being and the pleasure of living, aspects that have reached us to our day, since, for the very first time, they showed the house from a new perspective: that of the user.

As we have seen, the influence of these American female engineers is key to understand the advances in May's and Schütte-Lihotzky's modernist, European, social housing schemes. The echoes of the ideas of those pioneers still reverberates tenaciously in many aspects, ranging from the type of city they aspired to, to the normalized definition of the very last detail of design. The novelty has been the discovery of the unique traces their work has left behind and, most importantly, the way in which these advances reached May's group through women's associations. These organizations presented the different American trends to Lihotzky and infused in her the idea that domestic conditions had to be subjected to the same kind of rational scrutiny applied to the optimization of production. This is how rationalization and liberation were taken to the same level in Grete's mind.

During the interwar period, the everyday, the ordinary considerations that appeared in these women's manuals were adopted naturally in the design of collective housing. In this sense, women may be considered the originators of mass and popular culture ${ }^{36}$. Moreover, the kitchen may be considered, in a metaphorical sense, the epicenter of popular banality in what was then an emerging consumer culture. In the realm of the arts the influence exerted by lowbrow culture on highbrow culture came earlier and was more common, but in architecture this was the first time such leverage became evident. Over the past two centuries, but perhaps less so nowadays, women have taken on the role as the manufacturers and receptors of minor arts linked to subjective emotions and romantic passivity. There were certain trends in modernity that, in a sense, renounced mass culture, distancing themselves from all trivial and banal aspects of everyday life, taking refuge in aristocratic idealism and therefore cut off from the pulse of the ordinary. The universal adscription of femininity to media culture has always depended on its actual exclusion from high culture and its establishment.

A new path can be discovered thanks to these researchers, one that is upright but irreverent, prejudice free and that prioritizes the value of proximity, immediacy and lasting instants. The importance of domesticity, of the everyday, enables designers to think more about life within spaces than on the outer appearance of buildings. Besides, being as it is an intimate aspiration of human beings, the house is ascribed to publicity and consumer culture, midway between

p.72 technical progress and life style changes ${ }^{37}$. The contemporary role of women, their global relevance and their feminine expression, inevitably associated with domesticity, has displaced design concerns from the exterior of buildings to their interior. To us it is clear that the validity of domesticity through processes of use $\mathrm{e}^{38}$ and the household objects ${ }^{39}$ of the "ladies" we have mentioned persists in our way of understanding inhabitation, with their will to find happiness by means of a certain notion of domesticity that tried to reconcile traditional models with modern advances, advantages and comfort.

1. GROPIUS, Walter. Die Kunst in Industrie und Handel. Jena: Verlegt bei Eugen Diederichs, 1913.

2. BEHNE, Adolf. Der Moderne Zweckbau. Vienna/Berlin: Drei Masken Verlag, 1923.

3. LE CORBUSIER-SAUGNIER. Vers une architecture. Paris: Les Editions G. Cres et Cie., 1923.

4. GINZBURG, Moisei. Stil'i epokha. Moskva: Gosizdat, 1924.

5. MENDELSOHN, Erich. Amerika: Bilderbuch eines Architekten. Berlin: Rudolf Mosse Buchverlag, 1926

6. MCLEOD, Mary. 'Architecture or Revolution: Taylorism, Technocracy, and Social Change'. In: Art Journal, vol. 43, n. ${ }^{\circ}$ 2, pp. $132-147$.

7. NOEVER, Peter (ed.). Margarete Schütte-Lihotzky. Soziale Architektur Zeitzeugin eines Jahrhunderts. Wien: Bóhlau, 1993.

8. Ernst May und das neue Frankfurt 1925-1930, Catalogue of the Exhibition at the Deutschen Architekturmuseums, Frankfurt am Main. Berlin: Ernst \& Sohn, 1986.

9. BEECHER, Catharine. A Treatise on Domestic Economy, for the Use of Young Ladies at Home and at the School. Boston: Marsh, Capen, Lyon and Webb, 1841.

10. BEECHER, Catharine y BEECHER STOWE, Harriet. The American Woman's Home or Principles of Domestic Science Being a Guide to the Formation and Maintenance of Economical Healthful Beautiful and Christian Homes. New York: J. B. Ford and Company, 1869.

11. Known for having written Uncle Tom's Cabin or Life Among the Lowly in 1852.

12. Catharine Beecher had a delicate health and was always concerned with the correct ventilation of houses. Hence her insistence on the design of dynamic airing systems for each room. As an example of this gradual mechanization of the house, the book also includes many patents for innovative solutions. Her method entailed the search for an efficiency that guaranteed maximum health standards.

13. Even the drawings recall Diderot's encyclopedic graphics and his enlightened taxonomy where everything is articulated and disassembled in order to acquire prejudice-free knowledge.

14. FREDERICK, Christine. Selling Mrs. Consumer. New York: The Business Bourse, 1929

15. FREDERICK, Christine. The New Housekeeping: Efficiency Studies in Home Management. Garden City (New York): Doubleday, Page \& Company, 1913.

16. FREDERICK, Christine. Household Engineering: Scientific Management in the Home. Chicago: American School of Home Economics, 1920. 
17. GILBRETH, Lillian. The Psychology of Management: The Function of the Mind in Determining, Teaching and Installing Methods of Least Waste. New York: Sturgis \& Walton Company, 1914

18. GILBRETH, Frank B. y GILBRETH, Lillian. Fatigue Study: The Elimination of Humanity's Greatest Unnecessary Waste. A First step in Motion Study. New York: Sturgis \& Walton Company, 1916.

19. Filmed between 1910 and 1924, they were sponsored by the Chicago Chapter of the Society for the Advancement of Management.

20. Lillian Gilbreth studied English Literature at the University of California, Berkley, graduating in 1900. After that she decided to get a Masters degree in Psychology from Columbia University in New York. Even though she took doctoral courses in Berkley during 1911 in Psychology of Management, she did not get her PhD due to gender prejudices. This made her attain another PhD from Brown University in Providence, Rl, under the title Some Aspects of Eliminating Waste in Teaching.

21. TAUT, Bruno. Die Neue Wohnung. Die Frau als Schöpferin. Leipzig: Klinkhardt \& Biermann, 1924, p. 14 and pp. 65-66.

22. MEYER, Erna. Der neue Haushalt ein Wegweiser zu wirtschaftlicher Haushaltsführung. Stuttgart: Franckh'sche Verlagshandlung, 1926.

23. BOOT, Marjan y CASCIATO, Maristella (ed.) La casalinga riflessiva. La cucina razionale come mito domestico negli anni '20 e '30. Catalog of the Exhibition opened between October and November 1983 in the roman Palazzo delle Esposizioni. Roma: Multigrafica Editrice, 1983.

24. With the title Le Taylorisme chez soi. Pratique de la direction de la maison (Paris: Dunod Editeur, 1920) Frederick's first manual was published in France. The following year, it was published in German under the title Die rationelle Haushaltführung. Betriebswissenschaftliche Studien (Berlin: Julius Springer Verlag, 1921), translated by Irene Witte. It was also translated into Polish as Naukowa organizacja w gospodarstwie domowen (Warsaw: Nakladem Instytuta Naukowej Organizacji, 1926), with a prologue and an epilogue by Henry Le Chatelier, a well-known scientist who promoted industrial development. The Dutch version, published with the title De Denkende Huisvrouw, Nieuwe Inzichten (The Thinking Housewife, New Insights) (Haarlem: Tjeenk Willink, 1928), has an introduction by E.J. van Waveren-Resink, an activist and member of the Dutch Housewife Association. Lastly, Lorenzo Tealdy's italian translation was titled La donna e la casa. II taylorismo della vita domestica (Torino: C. Accame, 1928).

25. We have evidences of her dissertations in seven European countries: England, France, Belgium, Holland, Italy, Germany and Switzerland.

26. In 1920 she gave nine conferences, with the simultaneous translation of Irene Witte. Likewise, in 1924, she participated in the first Motion Study course in Europe, in Prague, and in the First International Management Congress. Meanwhile, in 1925, she participated in the IRI Congress in Vlissingen (the Netherlands), where she provided most of the scientific terms used for this matter.

27. Frank B. y Lillian M. Gilbreth: Ermüdungsstudium (Fatigue Study). Eine Einführung in das Gebiet des Bewegungsstudiums. Verlag des Vereines Deutscher Ingenieure. Berlin, 1921. Lillian Gillbreth: Verwaltungspsychologie. Berlin: Verl. d. Vereins dt. Ingenieure, 1922.

28. BERNĖGE, Paulette. 'Vers une cuisine automatique'. In: Mon Chez Moi. Installment IV, October 11923 - November 1 1923, pp. 25-29.

29. Regarding this, see HAYDEN, Dolores. The Grand Domestic Revolution: A History of Feminist Designs for American Homes, Neighborhoods, and Cities. Cambridge (Massachusetts): MIT Press, 1981.

30. Beecher y Beecher Stowe, op. cit., supra, note 10, p. 455.

31. In their text, the Beechers refer to the compact kitchens of steamboats. Íbidem, p. 32.

32. Ernst May: DAS NEUE FRANKFURT: Fünf Jahre Wohnungśbau in Frankfurt am Main. Frankfurt: Henrich Editionen, 2011. Installment of the international review Das Neue Frankfurt.

33. KAES, Anton; JAY, Martin and DIMENDBERG, Edward. The Weimar Republic Sourcebook: "Grete Lihotzky. Rationalization in the Household", University of California Press, Berkeley, 1994, pp. 462-465.

34. The title of the broadcasted conference was “Arbeitssparende Haushaltsführung”. NOEVER, Peter (ed.). op. cit., supra, nota 7, p. 75.

35. Directed by Paul Wolff in 1928, the movie is divided into four parts: The minimum dwelling, The Frankfurt kitchen, A new way of building and The prefabricated house. 36. ECO, Umberto. Apocalypse Postponed. Bloomington and Indianapolis: Indiana University Press, 1994.

37. ELSAESSER, Thomas. 'The Camera in Kitchen. Grete Schütte-Lihotzky and Domestic Modernity'. In: Christiane SCHÖNFELD, ed. and Carmel FINNAN. Practicing Modernity: Female Creativity in the Weimar Republic. Würzburg: Königshausen \& Neumann, 2006, pp. 27-49.

38. ECO, Umberto. The infinity of lists: An Illustrated Essay. New York: Rizzoli, 2009.

39. BAUDRILLARD, Jean. Le système des objets. Paris: Éditions Gallimard, 1969. 


\section{Autor imagen y fuente bibliográfica de procedencia}

Información facilitada por los autores de los artículos:

página 18, 1 (Crédito fotográfico gentileza de Federico Cairoli); página 19, 2 y página 20, 3 (Crédito fotográfico José Luis Uribe Ortiz); página 21, 4 (Crédito fotográfico gentileza de Berenice Gómez Crosa), 5 (Crédito boceto detalle constructivo gentileza de Luis Elgué); página 22, 6 (Crédito fotográfico gentileza de Estudio Elgué), 7 (Crédito boceto detalle constructivo gentileza de Lukas Fuster); página 23, 8 y 9 (Crédito fotográfico gentileza de Federico Cairoli), 10 (Crédito boceto detalle constructivo gentileza de José Cubilla); página 24, 11 y 12 (Crédito fotográfico gentileza de Federico Cairoli); página 29, 1 (Biblioteca Nacional de Australia. P490/7. 1918); página 30, 2, página 31, 3 y página 32, 4 (Javier Mosquera González); página 33, 5 (Biblioteca Nacional de Australia. PIC/9929/2029 LOC Cold Store PIC NICH), 6 (Biblioteca Nacional de Australia. PIC/9929/2110); página 34, 7 (Javier Mosquera González); página 35, 8 (Biblioteca Nacional de Australia. PIC/P2145); página 36, 9 (WikimediaCommons. Autor: Martyman); página 38, 10 (Javier Mosquera González); página 45, 1 (Elaboración propia); página 49, 2 (Elaboración propia, basado en información contenida en: MANNICHE, Peter, Living democracy in Denmark: independent farmers, farmer's cooperation, the folk high schools, cooperation in towns, social and cultural activities, social legislation, a Danish village. 2. ed. Copenhagen: G.E.C. Gad Pub., 1970. ISBN 0837139856), 3 (Elaboración propia, basado en la información contenida en: RUONAVAARA, Hannu. Home ownership and the Nordic housing policies in the 'Retrenchment phase'. En: Conference Building on Housing Ownership, Delft 2008; y en: TSENKOVA, Sasha; VESTERGAARD, Hedvig. Social Housing Provision in Copenhagen. Artículo presentado en ENHR 2011, Toulousse, France); página 50, 4 (Elaboración propia, a partir de la documentación original del proyecto aprobada por las autoridades de Copenhague, En base a la documentación cedida por Fællestegnestuen), 5 (Elaboración propia); página 51, 6 y 7 (Documentación cedida por Fællestegnestuen), 8 (HOLMBERG, Hartvig, ed. -indret selv Deres bolig. Copenhague: KAB, 1979, pp. 8-9); página 52, 9 (Documentación cedida por Fællestegnestuen), 10 (HOLMBERG, Hartvig, ed. -indret selv Deres bolig. Copenhague: KAB, 1979, portada y p. 43); página 53, 11 (HOLMBERG, Hartvig, ed. -indret selv Deres bolig. Copenhagen: KAB, 1979, p. 26), 12 (Elaboración propia); página 59, 1 (Parte superior: The American Woman's Home or Principles of Domestic Science Being a Guide to the Formation and Maintenance of Economical Healthful Beautiful and Christian Homes. New York: J. B. Ford and Company, 1869; The New Housekeeping: Efficiency Studies in Home Management. Garden City-New York: Doubleday, Page \& Company, 1913; The Psychology of Management: The Function of the Mind in Determining, Teaching and Installing Methods of Least Waste. New York: Sturgis \& Walton Company, 1914. Parte inferior: Catharine Beecher (Wikipedia Commons), Christine Frederick (Christine Frederick Archive, Schlesinger Library, Radcliffe Institute, Harvard University), Lillian Gilbreth (Lillian Moller Gilbreth Papers, Sophia Smith Collection, Northampton)); página 60, 2 y página 61, 3 (The American Woman's Home or Principles of Domestic Science Being a Guide to the Formation and Maintenance of Economical Healthful Beautiful and Christian Homes. New York: J. B. Ford and Company, 1869, p. 26, 37, 40); página 62, 4 (Christine Frederick Archive, Schlesinger Library, Radcliffe Institute, Harvard University); página 63, 5 (FREDERICK, Christine. The New Housekeeping: Efficiency Studies in Home Management. Garden City-New York: Doubleday, Page \& Company, 1913, p. 53); página 64, 6 (FREDERICK, Christine. Household Engineering: Scientific Management in the Home. Chicago: American School of Home Economics, 1920); página 65, 7 (Kheel Center for Labor-Management Documentation and Archives, Cornell University Library, Management Engineering), 8 (National Museum of American History, Behring Center, Division of Work and Industry Collection); página 66, 9 y 10 (Elaboración realizada por Carmen Espegel); página 67, 11 (Izquierda: BEECHER, Catharine y BEECHER STOWE, Harriet. The American Woman's Home or Principles of Domestic Science Being a Guide to the Formation and Maintenance of Economical Healthful Beautiful and Christian Homes. New York: J. B. Ford and Company, 1869, p. 23. Derecha: Siedlung Römerstad 1927-1928. Das Neue Frankfurt, n.4-5, Abril-Mayo, 1930, p. 76); página 68, 12 (Izquierda: HAYDEN, Dolores. The Grand Domestic Revolution: A History of Feminist Designs for American Homes, Neighborhoods, and Cities. Cambridge (Massachusetts): MIT Press, 1981, p. 30. Derecha: Ernst May und das Neue Frankfurt, 1925-1930. Catálogo de la exposición en el Deutsches Architekturmuseum Frankfurt am Main. Berlin: Wilhelm Ernst \& Sohn Verlag, 1986, p. 153); página 69, 13 (Superior izquierda y derecha: Restitución gráfica realizada por Carmen Espegel. Inferior izquierda: BEECHER, Catharine y BEECHER STOWE, Harriet. The American Woman's Home or Principles of Domestic Science Being a Guide to the Formation and Maintenance of Economical Healthful Beautiful and Christian Homes. New York: J. B. Ford and Company, 1869, pp. 28-30. Inferior derecha: Das Neue Frankfurt, n.6, Junio, 1929, p. 128), 14 (Izquierda: FREDERICK, Christine. Household Engineering: Scientific Management in the Home. Chicago: American School of Home Economics, 1920, p. 32. Derecha: Margarete SchütteLihotzky: Frankfurter Küche, 1926. Colección y Archivo de Margarete Schütte-Lihotzky en la Universidad de Artes Aplicadas de Viena); página 70, 15 (Izquierda: Original films of Frank and Lillian Gilbreth, promovida por Chicago Chapter, Society for the Advancement of Management, 1910-1924. San Francisco: National Film Preservation Foundation. Derecha: Neues Bauen in Frankfurt am Main, dirigida por Paul Wolff. Frankfurt: Wolff-Film, 1928); página 75, 1 (Enrique Jesús Fernández-Vivancos González a partir del plano de Tapiola de Aarne Ervi de 1963); página 76, 2 (Le Carré Bleu. 1960 nº 3); página 77, 3 y 4 (Museum of Finnish Architecture [MFA]); página 78, 5 (Le Carré Bleu.1958 nº 1 pp. 2-3. Fotógrafo: PIETINEN, Otson); página 79, 6 y 7, página 80, 8, 9 y 10 y página 81, 11 y 12 (Museum of Finnish Architecture [MFA]); página 82, 13 (Museum of Finnish Architecture [MFA]. Fotógrafo: LEHTONEN, Kai R); página 83, 14 (Museum of Finnish Architecture [MFA]); página 84, 15 (PIETILÄ, Reima. Noción Imagen Idea. Espoo: Teknillisen Korkeakoulun Ylioppilaskunta. 1975. Lección 5 y Lección 16); página 90, 1 (Natalia Matesanz Ventura); página 93, 2 y 3 (Fotograma del documental dirigido por Christina Holmes. 2015 [en línea] https://www.youtube.com/watch?v=bB-3Bp2pWh8); página 103, 1 (https://lebbeuswoods.files.wordpress.com/2011/06/Iw-mugshot1.jpg [en línea] [consulta: 22-08-2017]. Disponible en: https://lebbeuswoods.wordpress.com/2011/06/29/anti-journey-to-architecture-1/), 2 (https://lebbeuswoods.wordpress. com/2009/10/06/notebook-01-3-the-last/); página 106, 3 (https://lebbeuswoods.files.wordpress.com/2012/01/lbw-411.jpg[en línea] [consulta: 22-08-2017]. Disponible en: https://lebbeuswoods.wordpress.com/2012/01/02/origins/); página 108, 4 (https://lebbeuswoods.wordpress.com/2009/09/27/the-vagrant-light-of-stars/ [consulta: 22-08-2017]), 5 (https://lebbeuswoods.files.wordpress.com/2010/11/dwg-1.jpg [en línea] [consulta: 22-08-2017]. Disponible en: https://lebbeuswoods.wordpress. com/2010/11/02/drawings-stories/); página 109, 6 (https://lebbeuswoods.wordpress.com/2009/08/01/meta-institutes/ [consulta: 22-08-2017]); página 110, 7 (https:// lebbeuswoods.wordpress.com/2009/03/15/notebook-97-3/ [consulta: 22-08-2017]), 8 (https://lebbeuswoods.wordpress.com/2009/12/19/storm-watch/ [consulta: 2208-2017]); página 111, 9 (https://lebbeuswoods.wordpress.com/2009/06/05/architecture-of-energy/ [consulta: 22-08-2017]), 10 (https://lebbeuswoods.wordpress. com/2011/02/15/a-space-of-light-2/ [consulta: 22-08-2017]); página 112, 11 (https://lebbeuswoods.files.wordpress.com/2012/03/Itpav-may-30-3.jpg [en línea] [consulta: 22-08-2017]. Disponible en: https://lebbeuswoods.wordpress.com/2012/03/25/light-pavilion-under-construction/); página 113, 12 (https://lebbeuswoods.files.wordpress. com/2011/01/cdusk1day.jpg [en línea] [consulta: 22-08-2017] Disponible en: https://lebbeuswoods.wordpress.com/2011/02/15/a-space-of-light-2/); página 114, 13 (https://lebbeuswoods.wordpress.com/2009/09/19/line-up/ [consulta: 22-08-2017]), 14 (https://lebbeuswoods.files.wordpress.com/2011/02/int-5-11.jpg [en línea] [consulta: 22-08-2017]. Disponible en: https://lebbeuswoods.wordpress.com/2011/02/15/a-space-of-light-2/), 15 (Fotografía: Shu He. Imagen cedida por Steven Holl Architects) 